\title{
The Golden Age of Cataclysmic Variables and Related Objects: The State of Art
}

\author{
Franco Giovannelli*i \\ INAF - Istituto di Astrofisica e Planetologia Spaziali, Via del Fosso del Cavaliere, 100, 00133 \\ Roma, Italy \\ E-mail: franco.giovannellidiaps.inaf.it
}

\section{Lola Sabau-Graziati}

INTA- Dpt. Cargas Utiles y Ciencias del Espacio, C/ra de Ajalvir, Km 4 - E28850 Torrejón de Ardoz, Madrid, Spain

E-mail: sabaumdainta.es

\begin{abstract}
This paper is an extended and updated version of the reviews by Giovannelli (2008) and Giovannelli \& Sabau-Graziati (2015a). In this paper we review cataclysmic variables (CVs) discussing several hot points about the renewing interest of today astrophysics about these sources. We will briefly discuss also about classical and recurrent novae, as well as the intriguing problem of progenitors of the Type Ia supernovae.

However, the bulk of this paper consists in our attempt of studying CVs from the point of view of the magnetic field intensity (B) at the surface of their white dwarfs.

Because of limited length of the paper and our knowledge, this review does not pretend to be complete. However, we would like to demonstrate that the improvement on knowledge of the physics of our Universe is strictly related also with the multifrequency behaviour of CVs, which apparently in the recent past lost to have a leading position in modern astrophysics.
\end{abstract}

The Golden Age of Cataclysmic Variables and Related Objects - III, Golden2015

7-12 September 2015

Palermo, Italy

* Speaker.

${ }^{\dagger}$ A footnote may follow. 


\section{Introduction}

In the 1950s it was recognized that the various phenomena displayed by the CVs are all the consequence of accretion of matter onto a white dwarf (WD) from a low mass donor star (e.g., Warner, 1976; 1995a). CVs are binary systems in which the primary component is a WD $\left(M_{\mathrm{wd}} \sim 1\right.$ $\left.\mathrm{M}_{\odot}\right)$ and the secondary is a late type Main Sequence $\operatorname{star}\left(M_{\mathrm{s}} \leq 1 \mathrm{M}_{\odot}\right)$ (e.g., Smak, 1985a).

Mass transfer is strongly depending, besides the orbital parameter of the system, on the magnetic field intensity at the surface of the primary. Such process produces a large fan of behaviour that are detectable in different energy ranges: from radio to X-rays, and even in $\gamma$-rays. The orbital periods of $\mathrm{CVs}$ are ranging from $\sim 80 \mathrm{~m}$ to $\sim 12 \mathrm{~h}$ with a distribution showing a gap between 2 and 3 hours, in which few systems have been detected. In the past this gap was empty and this was the reason because was nicknamed 'period gap'.

HEASARC database (https://heasarc.gsfc.nasa.gov/W3Browse/all/rittercv.html) table contains information on cataclysmic binaries only, as taken from the Catalog of Cataclysmic Binaries, LowMass X-ray Binaries, and Related Objects (7th Edition, Release 7.21, March 2014) of Ritter \& Kolb. The complete catalog (Ritter \& Kolb, 2003, A\&A 404, 301) lists coordinates, apparent magnitudes, orbital parameters, stellar parameters of the components, and other characteristic properties of 1166 cataclysmic binaries, 105 low-mass X-ray binaries, and 500 related objects with known or suspected orbital periods. Most of CVs were discovered through optical observations, and some, especially those in which the magnetic field of the WD is strong, discovered through $\mathrm{X}$-ray observations, but with the detectors of the second and further generations, since CVs are in general not very bright in X-ray energy range.

The catalog of Downes et al. (2001, 2006) reports 1830 CVs. Adding the new discoveries (530 new CVs) made by the MASTER-NET experiment (Buckley et al., 2015), the number of CVs known is greater than 2000 .

The first CV detected in the X-ray range, with rocket experiments, was the dwarf nova SS Cyg (Rappaport et al., 1974; Heise et al., 1978). The UHURU satellite detected two CVs, which were not recognized as such. Warner (1976) proposed the identification of 4U 1249-28 with EX Hya, and the variable AM Her, which on further optical studies was recognized as a CV (Forman et al., 1978). The magnetic field in these two systems is strong $\left(\approx 10^{7}-10^{8} \mathrm{G}\right)$. A few dozen CVs were detected in X-rays with HEAO-1 satellite, with EXOSAT, and with the Einstein satellite (e.g., reviews of Cordova \& Mason, 1983; Cordova, 1995). Later Verbunt et al. (1997) recognized 91 CVs from a sample of 162 systems with known or suspected binary periods by using data of the ROSAT XRT-PSPC All Sky Survey.

Historically, because CVs were observed photometrically and without seeming to follow any regular pattern, they were named with the term cataclysmic (from the Greek word kataklysmos = flood, storm; Hack \& la Dous, 1993). As collecting of observational data progressed it became apparent that these objects were regular binary systems which for some reason changed in brightness; some of them also regularly (Recurrent Novae and Dwarf Novae) while some others only once (Classical Novae). Therefore the classification of CVs was based on the optical outburst properties, by which one may distinguish four groups of CVs: (i) classical novae; (ii) recurrent novae; (iii) dwarf novae; (iv) nova-like objects (e.g., Giovannelli \& Martinez-Pais, 1991 and references therein; Ritter, 1992; Giovannelli, 2008). This classification, however, is neither self-consistent 
nor adequate and it is much better to consider primarily the observed accretion behaviour (Smak 1985b). One obvious advantage of such an approach is connected with the time scales of various accretion phenomena, which are sufficiently short to avoid any major observational bias: the mass accretion rates in CVs usually range from $10^{-11}$ to $10^{-8} \mathrm{M}_{\odot} \mathrm{yr}^{-1}$ (Patterson, 1984); the time scales are from tens of seconds (oscillations in dwarf novae at outbursts) to years (super-outbursts of SU UMa stars or long term variations in VY Scl stars).

However, in the class of nova-like objects there are two sub-classes: the DQ Her stars and the AM Her stars. In these sub-classes of CVs the WDs possess magnetic fields with intensity enough high for dominating the accretion disk and all the phenomena related to it. These classes of magnetic CVs, whose names are coming from the prototypes DQ Her and AM Her took later the names of Intermediate Polars and Polars, respectively. A short history of their discovery has been discussed by Warner (1995b). Fundamental papers about these sub-classes are those by Patterson (1994), Warner (1996). The class of IPs has been split into two subclasses with relatively large and relatively weak magnetic field (Norton et al., 1999). One example of a system belonging to the latter subclass is DO Dra (previously registered as YY Dra) (Andronov et al., 2008).

There is another class of CVs, the rare AM Canum Venaticorum (AM CVn) star systems. They have extremely short orbital periods between $\sim 10-65$ minutes. Their spectra do not show evidence for hydrogen. They appear to be helium-rich versions of CVs (e.g. Warner, 1995c; Nelemans, 2005). There is an old suggestion, that in these systems the mass transfer is driven by gravitational wave radiation losses, proposed by Paczyński (1967), after the discovery of the prototype with an orbital period of $\sim 17$ minutes (Smak, 1967).

\section{The role of magnetic field in cataclysmic variables}

Depending on the magnetic field intensity at the WD, the accretion of matter from the secondary star onto the primary can occur either via an accretion disc (in the so-called Non-Magnetic CVs: NMCVs) or a channelling through the magnetic poles (in the case of Polars: PCVs) or in an intermediate way (in the case of Intermediate Polars: IPCVs).

$\mathrm{CVs}$ in a time scale of order between weeks and years flare up almost periodically, about few magnitudes in optical wavelengths; the duration of the outbursts is much shorter than the recurrence time. Typical light curves for classical novae and dwarf novae of the U Gem, Z Cam, and SU UMa types can be seen in Ritter (1992) and e.g. in Giovannelli (2008).

The recurrence time-scale of outbursts in dwarf novae is correlated with their amplitude and the outburst duration is depending on the orbital period (Warner, 1987).

A recent paper by Otulakowska-Hypka, Olech \& Patterson (2016) present a statistical study of all measurable photometric features of a large sample of dwarf novae during their outbursts and superoutbursts. They used all accessible photometric data for all their objects to make the study as complete and up to date as possible. Their aim was to check correlations between these photometric features in order to constrain theoretical models which try to explain the nature of dwarf novae outbursts. They managed to confirm a few of the known correlations, that is the Stolz and Schoembs relation, the Bailey relation for long outbursts above the period gap, the relations between the cycle and supercycle lengths, amplitudes of normal and superoutbursts, amplitude and duration of superoutbursts, outburst duration and orbital period, outburst duration and mass 
ratio for short and normal outbursts, as well as the relation between the rise and decline rates of superoutbursts. However, they question the existence of the Kukarkin-Parenago relation but they found an analogous relation for superoutbursts. They also failed to find one presumed relation between outburst duration and mass ratio for superoutbursts. This study should help to direct theoretical work dedicated to dwarf novae.

In PCVs the WD magnetic field is strong enough to make the Alfvén radius greater than the circularization radius, so no accretion disk is formed and the accretion structure is fully governed by the magnetic field, which canalize the accreting matter across the field lines. Owing to the intense magnetic field ( 10-200 MG), the WD rotation is synchronized with the binary orbital period (a few hours). However, there are few systems (V1432 Aql, BY Cam, V1500 Cyg, V4633 Sgr, and CD Ind) in which $\mathrm{P}_{\text {spin }}$ and $\mathrm{P}_{\text {orb }}$ differ by around $2 \%$ or less. These are assumed to be polars that have been disturbed from synchronism by a recent nova explosion (Norton, Sommerscales \& Wynn, 2004, and the references therein).

Boyd et al. (2014) reported the results of a 15-year campaign by the globally distributed Center for Backyard Astrophysics to observe V1432 Aql - the only known eclipsing asynchronous polar and investigate its return to synchronism. Originally knocked out of synchrony by a nova explosion before observing records began, the magnetic white dwarf in V1432 Aql is currently rotating slower than the orbital period but is gradually catching up. At the present rate, synchronism should be achieved around 2100. The continually changing trajectory of the accretion stream as it follows the magnetic field lines of the rotating white dwarf produces a complex pattern of light emission that Boyd et al. (2014) have measured and documented, providing comprehensive observational evidence against which physical models of the system can be tested.

IPCV WDs have moderate magnetic fields (order of a few MG); the Alfvén radius is smaller than the circularization radius but it is greater than the WD radius. Therefore an accretion disk is formed in these systems but being disrupted at its inner region. In IPCVs matter follows again the magnetic field lines but just inside the Alfvén radius. The rotating WD is asynchronous with the binary orbital period $\left(\mathrm{P}_{\text {spin }} \ll \mathrm{P}_{\text {orb }}\right)$. However, there are few systems that may be best described as nearly synchronous intermediate polars (V381 Vel, RXJ0524+42, HS0922+1333, and V697 Sco) (Norton, Sommerscales \& Wynn, 2004, and the references therein). Two of these systems lie in the 'period gap'. Probably all four systems are IPs in the process of attaining synchronism and evolving into polars.

The last group defined by the accretion structure criterion, NMCVs, includes those systems whose WD magnetic fields are not relevant in governing the accretion structure. In these systems the accretion disk extends down to the WD surface and a boundary layer is formed. This family shows a great diversity of observational behaviour; for this reason the historical criterion of classification is, in this case, more appropriate for distinguishing their sub-classes. However, it is simply an attempt of classification for lack of a more general physical classification (e.g., Giovannelli, 1991 and references therein). Indeed, in general, we can consider the WD of a CV as a gravimagnetic rotator, characterized by a mass $\mathrm{M}$ - accreting matter at a rate $\dot{\mathrm{M}}$ from the optical companion (the secondary star) — rotating with a velocity $\vec{\omega}$ and having a magnetic moment $\vec{\mu}$, not necessarily coaxial with the rotational axis (Lipunov, 1987; 1991). Then the accreting system is completely characterized by the following physical parameters: mass $\mathrm{M}$, accretion rate $\dot{\mathrm{M}}$, rotational velocity $\vec{\omega}$, and magnetic moment $\vec{\mu}$. In the plane spin period of the WD (and in general of the compact 
object) - gravimagnetic parameter $\mathrm{y}=\dot{\mathrm{M}} / \mu^{2}$ it is possible to find any sort of physical conditions of gravimagnetic rotators, as discussed by e.g. Giovannelli (1991).

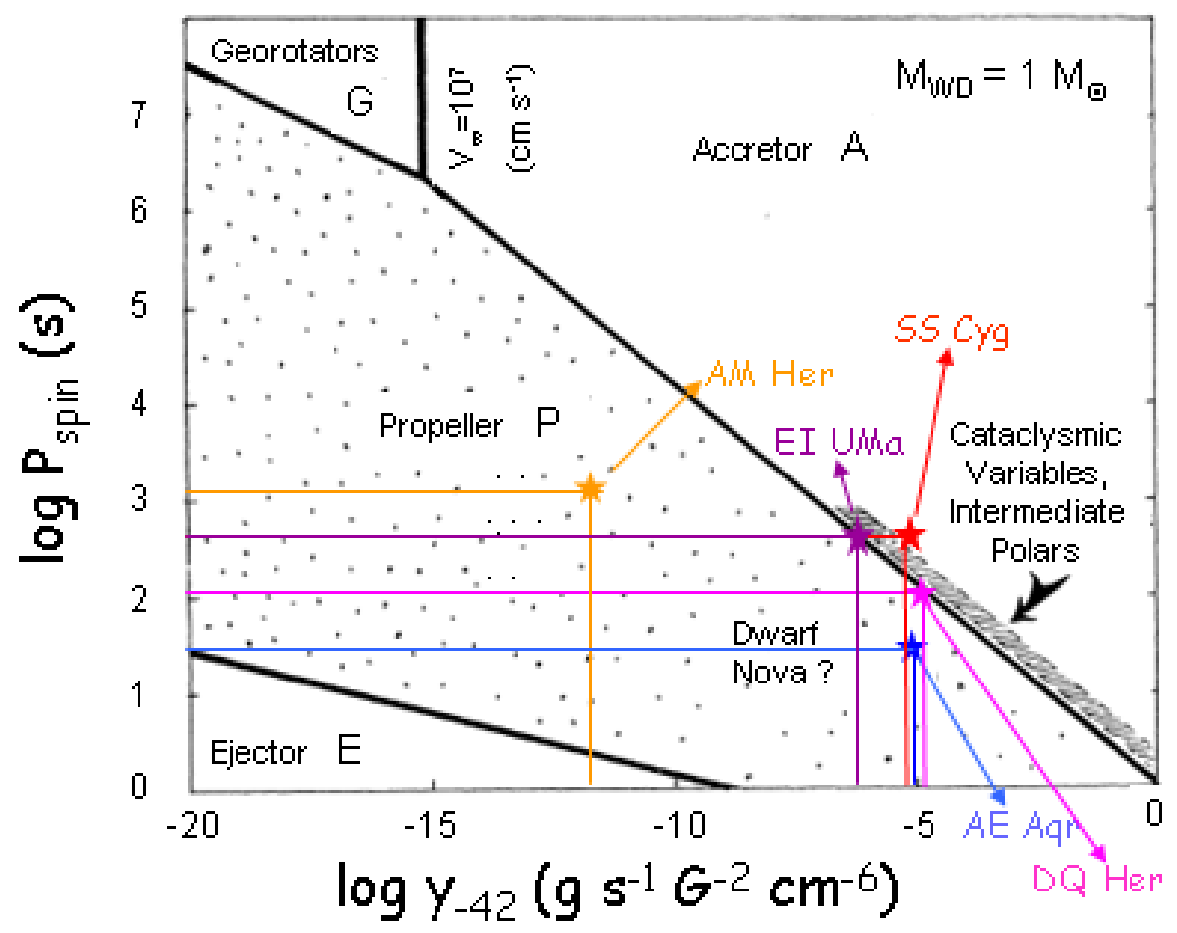

Figure 1: The positions of several CVs in Lipunov's diagram calculated for $1 \mathrm{M}_{\odot}$ white dwarf (after Lipunov, 1987).

Figure 1 shows the diagram spin period of the WD versus gravimagnetic parameter, where $\mathrm{P}_{\text {spin }}$ is expressed in seconds and the gravimagnetic parameter is expressed in unit of $10^{-42} \mathrm{~g}$ $\mathrm{s}^{-1} \mathrm{G}^{-2} \mathrm{~cm}^{-6}$. This diagram shows the positions of few well known CVs: AM Her (Terada et al., 2010 and references therein), AE Aqr (Patterson, 1979; de Jager et al., 1994; Wynn, King \& Horne, 1997), DQ Her (Patterson, 1994; Zhang et al., 1995), EI UMa (Reimer et al., 2008), SS Cyg (Giovannelli \& Sabau-Graziati 2012a). It appears evident the power of this diagram obtained by Lipunov (1987) using $1 \mathrm{M}_{\odot}$ white dwarf. The polars AM Her and AE Aqr lie in the zone of propeller, how they must stay, while the IPCV DQ Her (the prototype of this class), EI UMa (a very well known IPCV), and SS Cyg (whose nature as IPCV is claimed by Giovannelli's group on the base of many circumstantial proofs and also because of a cogent similarity with EI UMa, e.g. Giovannelli \& Sabau-Graziati 2012a,c) lie just in the zone predicted by Lipunov for such objects.

As recalled by Giovannelli \& Sabau-Graziati (1999), it is evident that the properties of an outburst in CVs depend crucially on the accretion rate, the mass of the WD, and the chemical composition of its hydrogen rich envelope in which the thermonuclear runaway occurs. And the accretion process onto the WD is strongly influenced by its magnetic field intensity. Indeed, the three kind of CVs (non-magnetic, polars, and intermediate polars) obey to relationships between the orbital period of the system and the spin period of the WD (Warner \& Wickramasinghe, 1991), where the magnetic field intensity plays a fundamental role. The orbital evolution of CVs, and hence the mass-transfer rate $(\dot{\mathrm{M}})$ from the secondary to the white dwarf is driven by magnetic 
braking of the secondary for long-period systems $\left(\mathrm{P}_{\mathrm{orb}}>3 \mathrm{hr}\right)$ and gravitational radiation for shortperiod systems $\left(\mathrm{P}_{\text {orb }}<2 \mathrm{hr}\right)$.

However, such a gap - which was believed true for long time - is now partially filled by the SW Sex systems (e.g. Rodriguez-Gil, 2003; Rodriguez-Gil et al., 2007). The apparent 'period gap' was due to a smaller number of systems having orbital periods in such an interval, which were escaping from the observations.

Therefore, the role of magnetic field intensity plays a fundamental role in the process of accretion of matter onto the compact star. Therefore Lipunov's diagram ( $\left.\log \mathrm{P}_{\text {spin }} \mathrm{vs} \log \mathrm{y}\right)$ appears as the best way for localizing the position of MWDs, both polars and IPs, as shown in Fig. 1.

It is evident that a fundamental parameter characterizing the whole sample of CVs is the orbital period, which is strictly connected with the evolution of the systems. Fig. 2 shows the number of CVs versus the orbital period (after Gänsicke, 2005). Below and above the so-called "period gap" $\sim 39 \%$ and $50 \%$ of CVs lie, respectively; about $11 \%$ of CVs lie within the "period gap" which is partially filled by the zone where the SW Sextantis systems lie (e.g. Rodriguez-Gil, 2003). Howell, Nelson \& Rappaport (2001) discussed critically the basic paradigm for the origin of the 2-3 $\mathrm{hr}$ "period gap" in CVs.

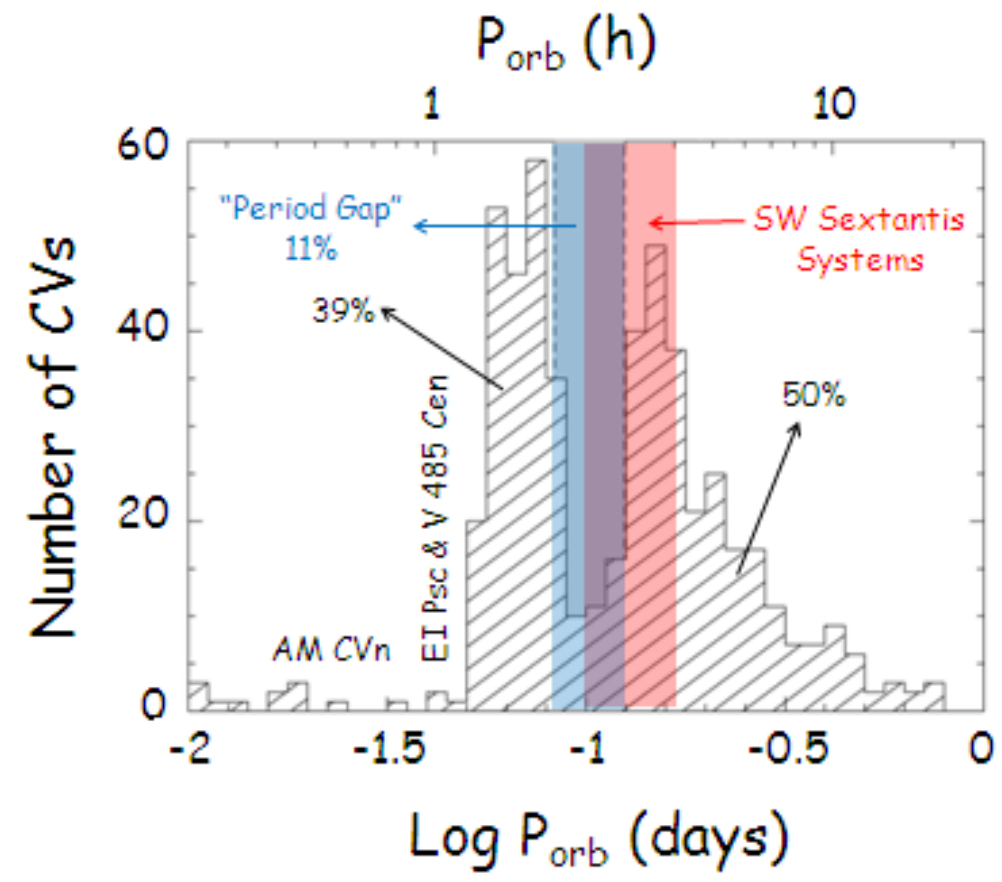

Figure 2: Number of CVs versus orbital period. Light blue rectangle shows the so-called "period gap"; light red rectangle shows the range of periods where SW Sextantis systems lie. Cyan-50 rectangle represents the intersection between the "period gap" and SW Sex periods (after Gänsicke, 2005 and Rodriguez-Gil, 2003).

Accreting binaries with white dwarf primaries and main sequence secondaries have binary orbital periods greater than $80 \mathrm{~min}$. For shorter period systems the secondary must be degenerate or semi-degenerate: e.g. white dwarf - white dwarf binaries.

The shorter period AM Canum Venaticorun (AM CVn) systems lie well below the "period 
gap". The prototype HZ $29=$ AM CVn, with $\mathrm{P}_{\text {orb }} \sim 17.5$ min, was discovered by Smak (1967) and was recognized as a CV by Patterson (1992). Nelemans (2005) published a short review about "AM CVn Stars" and later a review about "AM CVn Stars: Status and Challenges" was published by Solheim (2010). The importance of such systems is remarked in the study of interacting double white dwarf binaries that can give rise to a wide variety of astrophysical outcomes ranging from faint thermonuclear and Type-Ia supernovae (SNe Ia) to the formation of neutron stars and stably accreting AM CVn systems. One key factor affecting the final outcome is whether mass transfer remains dynamically stable or instead diverges, leading to the tidal disruption of the donor and the merger of the binary. It is typically thought that for low ratios of the donor mass to the accretor mass, mass transfer remains stable, especially if accretion occurs via a disk. Shen (2015) examines low mass ratio double WD binaries and finds that the initial phase of hydrogen-rich mass transfer leads to a classical nova-like outburst on the accretor. Dynamical friction within the expanding nova shell shrinks the orbit and causes the mass transfer rate to increase dramatically above the accretor's Eddington limit, possibly resulting in a binary merger. If the binary survives the first hydrogen-rich nova outbursts, dynamical friction within the subsequent helium-powered nova shells pushes the system even more strongly toward merger.

And the merging process of two compact stars has recently become extremely important for the gravitational wave $(\mathrm{GW})$ astronomy. It is necessary to explore with particular attention each signal coming from GW detectors, possibly with multifrequency observations, in all the possible ranges, triggered by the $\mathrm{GW}$ detection.

Kalomeni et al. (2016) present a binary evolution study of CVs and related systems with white dwarf accretors, including for example, AM CVn systems, classical novae, supersoft X-ray sources, and systems with giant donor stars. They indicate where in the relationship $\mathrm{P}_{\text {orb }}-\mathrm{M}_{\text {donor }}$ the accretion disks will tend to be stable against the thermal-viscous instability, and where gravitational radiation signatures may be found with LISA.

Cannizzo \& Nelemans (2015) use the observed range of outbursting behavior for AM CVn systems as a function of orbital period to place a constraint on mass transfer rate versus orbital period. They infer a rate $\sim 5 \times 10^{-9} \mathrm{M}_{\odot} \mathrm{yr}^{-1} \times\left(\mathrm{P}_{\text {orb }} / 1000 \mathrm{~s}\right)^{-5.2}$. This functional form obtained is consistent with the recurrence time - orbital period relation found by Levitan et al. (2015) using a simple theory for the recurrence time.

Therefore the investigation on the magnetic field intensities in WDs is crucial in understanding the evolution of CVs systems. The fundamental parameters to be searched are the magnetic moment, the mass accretion rate and the orbital parameters of the systems. In this way it will be possible to fulfill the plane $\log \mathrm{P}_{\text {spin }}-\log \mathrm{P}_{\text {orb }}$, where a priori there are not restricted ranges of magnetic moment $|\vec{\mu}|$, or special correlations between $\mathrm{P}_{\text {spin }}$ and $\mathrm{P}_{\text {orb }}$ and $|\vec{\mu}|$. The distribution of objects in that diagram is owed to the interaction of braking torques and accretion torques, with the superposition of the observed or implied variations of the accretion rate on long time scale $\left(>10^{2}\right.$ yr), acting on a continuum of magnetic moments. In this way each system is completely described by those physical parameters.

Davis et al. (2008) applied population synthesis techniques to calculate the present day number of two types of WD-main sequence star (WDMS) binaries within the 'period gap'. The first are post-common envelope binaries with secondary stars that have masses $0.17 \leq \mathrm{M}_{\mathrm{s}} / \mathrm{M}_{\odot} \leq 0.36$ (gPCEBs), such that they will commence mass transfer within the period gap. The second type are 
systems that were CVs at some point in their past, but detached once they evolved down in orbital period to $\approx 3 \mathrm{~h}$ as a consequence of disrupted magnetic braking, and are crossing the 'period gap' via gravitational radiation (dCVs). They predicted an excess of dCVs over gPCEBs within the 'period gap' of $\sim 4$ to $\sim 13$. This excess is revealed as a prominent peak at the location of the 'period gap' in the orbital period distribution of the combined gPCEB and dCV populations. They suggest that if such a feature is observed in the orbital period distribution of an observed sample of short orbital period WDMS binaries, this would strongly corroborate the disruption of magnetic braking.

Willems et al. (2005) and Willems et al. (2007) by using population synthesis tools studied the population of NMCVs with orbital periods 1$)<2.75 \mathrm{~h}$, and 2) $>2.75 \mathrm{~h}$, respectively.

1) A grid of detailed binary evolutionary sequences was calculated and included in the simulations to take account of additional angular momentum losses beyond that associated with gravitational radiation and mass loss, due to nova outbursts, from the system. As a specific example, Willems et al. (2005) considered the effect of a circumbinary disk to gain insight into the ingredients necessary to reproduce the observed orbital period distribution. The resulting distributions showed that the period minimum lies at about 80 minutes, with the number of systems monotonically increasing with increasing orbital period to a maximum near 90 minutes. There is no evidence for an accumulation of systems at the period minimum, which is a common feature of simulations in which only gravitational radiation losses are considered. The shift of the peak to about 90 minutes is a direct result of the inclusion of systems formed within the period gap.

2) The population of NMCVs with unevolved main-sequence-like donors at orbital periods greater than $2.75 \mathrm{~h}$ was investigated. In addition to the angular momentum losses associated with gravitational radiation, magnetic braking, and mass loss from the system, Willems et al. (2007) also included the effects of circumbinary disks on the evolution. For a fractional mass input rate into the disk, corresponding to $3 \times 10^{-4}$ of the mass transfer rate, the model systems exhibit a bounce at orbital periods greater than $2.75 \mathrm{hr}$. The simulations revealed that: i) some systems can exist as dwarf novae throughout their lifetime, ii) dwarf novae can evolve into novalike systems, and iii) novalike systems can evolve back into dwarf novae during their postbounce evolution to longer orbital periods. Among these subclasses, novalike cataclysmic variables would be the best candidates to search for circumbinary disks at wavelengths $\geq 10 \mu \mathrm{m}$. The theoretical orbital period distribution is in reasonable accord with the combined population of dwarf novae and novalike systems above the period gap, suggesting the possibility that systems with unevolved donors need not detach and evolve below the period gap as in the disrupted magnetic braking model. Experimental data are necessary for checking the validity of theoretical predictions.

The field strength distribution of MCVs $\left(\sim 10^{5}-10^{9} \mathrm{G}\right)$ differs from that of single MWDs $\left(\sim 10^{3}-10^{9} \mathrm{G}\right)$ (Ferrario, de Martino \& Gänsicke, 2015), although both cluster around $30 \mathrm{MG}$. While the upper limit cutoff near $10^{9} \mathrm{G}$ appears to be real, the lower limit is more difficult to investigate. The incidence of magnetism below a few $10^{3} \mathrm{G}$ still needs to be established by sensitive spectropolarimetric surveys conducted on $8 \mathrm{~m}$ class telescopes.

In accreting WDs of CVs, as far as is presently known, there is a lack of systems at both high and low field strengths. However, the apparent absence of low field MCVs might be explained by the IPs, which generally have unknown field strengths, and the lack of high field systems, though possible, is still not understood (e.g., Beuermann, 1998). Wynn (2000) discussed the problem of accretion flows in MCVs. On the base of the ratio $\mathrm{P}_{\text {spin }} / \mathrm{P}_{\text {orb }}$ he divided the MCVs in three 
classes: class 1 , class 2 and class 3 if such a ratio is $\ll 0.1, \sim 0.1$, and $\gg 0.1$, respectively. For the systems in class 1 the disk equilibrium condition is clearly satisfied. Those in class 2 are very unlikely to possess accretion disks. The systems in class 3 are EX Hya-like systems which lie below the 'period gap' and cannot possibly contain accretion disks. These are EX Hya, HT Cam, RXJ1039.7-0507, and V1025 Cen. These all have $\mathrm{P}_{\text {spin }} / \mathrm{P}_{\text {orb }}>0.1$ and $\mathrm{P}_{\text {orb }}<2$ hr. DD Cir and V795 Her lie within the 'period gap' with $\mathrm{P}_{\text {spin }} / \mathrm{P}_{\text {orb }} \sim 0.1$ and may be included in the class 2 (Norton, Somerscales \& Wynn, 2004, and the references therein). Wynn (2000) crudely classified the MCVs according to the magnetic moment and orbital period. EX Hya systems have magnetic moment similar to IPs above the 'period gap' and comparable to the weakest field AM Her-like systems. This indicates that MCVs above the 'period gap' will evolve to long spin periods below it. Norton, Wynn \& Somerscales (2004) investigated the rotational equilibria of MCVs. They predicted that IPCVs with $\mu \geq 5 \times 10^{33} \mathrm{G} \mathrm{cm}^{3}$ and $\mathrm{P}_{\text {orb }}>3 \mathrm{hr}$ will evolve into PCVs, whilst those with $\mu \leq 5 \times 10^{33} \mathrm{G} \mathrm{cm}^{3}$ and $\mathrm{P}_{\text {orb }}>3 \mathrm{hr}$ will either evolve into low field strength polars that are presumably unobservable, and possibly EUV emitters, or into PCVs when their fields, buried by high accretion rate, revive when the mass accretion rate reduces.

Warner (1996) deeply discussed torques and instabilities in IPs on the base of measured spin periods of the primaries and found several important relationships between fundamental parameters of these systems, such as $\log \dot{\mathrm{M}}$ vs $\log \mathrm{P}_{\text {orb }}, \log \mu_{33}$ vs $\log \dot{\mathrm{M}}_{17}, \log \mathrm{L}_{\mathrm{X}}$ vs $\log \dot{\mathrm{M}}$, as shown in Fig. 3 in the left, central, and right panels, respectively. There is a range of magnetic moments $\mu$ and mass transfer rates in which synchronized rotation of the primary can occur even though it possesses an accretion disk.

Ak et al. (2010), using available astrometric and radial velocity data, computed the space velocities of CVs with respect to the Sun and investigated kinematical properties of various subgroups of CVs. The orbital period distribution of CVs in the refined sample of 159 systems resembles that of the whole sample of CVs (e.g. Connon Smith, 2007). Ak et al. (2010) found that the mean kinematical age (MKA) of the 159 systems is $\mathrm{MKA}_{159}=5 \pm 1$ Gyr. In the sample, 134 of 159 systems are non magnetic $(\mathrm{NMCV})$ having $\mathrm{MKA} \mathrm{NMCV}_{\mathrm{N}}=4.0 \pm 1.0 \mathrm{Gyr}$. In the sub-sample of NMCVs, 53 of 134 have $\mathrm{P}_{\text {orb }}<2.62 \mathrm{~h}$ and their MKA is $5.0 \pm 1.5 \mathrm{Gyr}$, whilst 81 of 134 systems have $\mathrm{P}_{\text {orb }}>2.62 \mathrm{~h}$ and their MKA is $3.6 \pm 1.3 \mathrm{Gyr}$. This means that CVs below the 'period gap' are older than systems above the gap. This results in agreement with the standard evolution theory of CVs. The selection of $2.62 \mathrm{~h}$ as the border between the two groups of systems lies roughly in the middle of the 'period gap', where systems have been detected. This means that the 'period gap' does not exist anymore and the systems inside this 'gap' are just frontier objects between systems experiencing gravitational radiation and those experiencing magnetic braking. The reason because they are not so numerous as those placed at sides could be the relative shorter time of permanence in the 'gap', and then difficult to be detected.

Following the interesting review by Ferrario, de Martino \& Gänsicke (2015), highly magnetic WDs (HMWDs) tend to exhibit a complex and non-dipolar field structure with some objects showing the presence of higher order multipoles. There is no evidence that fields of HMWDs decay over time, which is consistent with the estimated Ohmic decay times scales of $\sim 10^{11} \mathrm{yrs}$. The slow rotation periods ( $\sim 100 \mathrm{yrs}$ ) inferred for a large number of isolated MWDs in comparison to those of non-magnetic WDs (a few days) suggest that strong magnetic fields augment the braking of the stellar core. MWDs, as a class, also appear to be more massive $\left(0.784 \pm 0.047 \mathrm{M}_{\odot}\right)$ than 


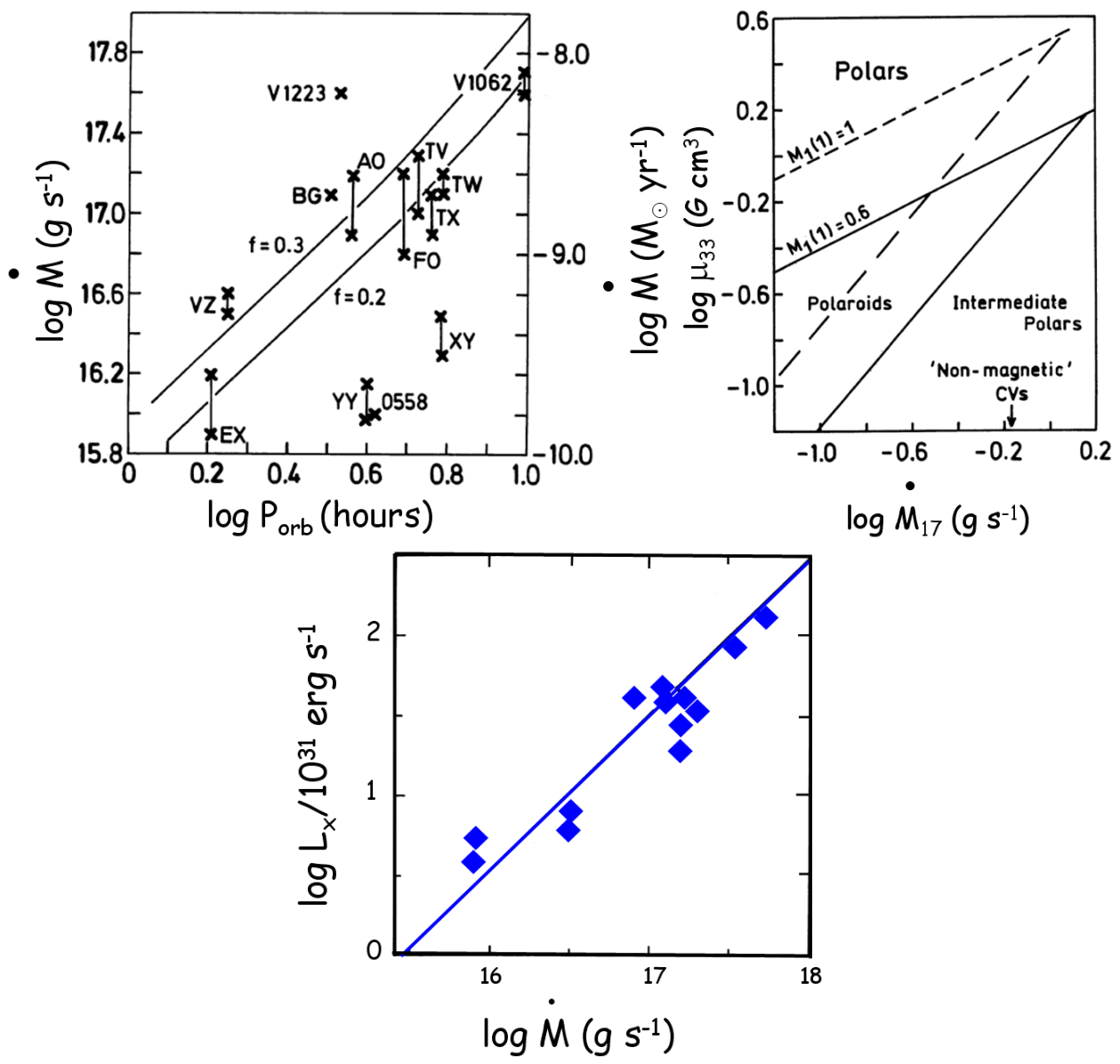

Figure 3: Left upper panel: Intermediate polars in the plane $\dot{\mathrm{M}}-\mathrm{P}_{\text {orb }}$. The two lines are lines of disc stability: stable above and dwarf nova outburst below. They were computed for $\mathrm{f}=0.2$ and 0.3 , with $\mathrm{R}_{\text {out }}=\mathrm{f} \times \mathrm{a}$, being a the separation of the two stars in the system (by courtesy of Warner, 1996). Right upper panel: Magnetic moment in units of $10^{33} \mathrm{G} \mathrm{cm}^{3}$ versus mass accretion rate in units of $10^{17} \mathrm{~g} \mathrm{~s}^{-1}$. Boundaries for white dwarf mass $\mathrm{M}_{1}=1 \mathrm{M}_{\odot}$ and $\mathrm{M}_{1}=0.6 \mathrm{M}_{\odot}$ have been computed for $\mathrm{P}_{\text {orb }}=4 \mathrm{~h}$ (by courtesy of Warner, 1996). Lower panel: Mass transfer rate onto white dwarf versus (2-10 keV) X-ray luminosity (by courtesy of Warner, 1996).

their weakly or non-magnetic counterparts $\left(0.663 \pm 0.136 \mathrm{M}_{\odot}\right)$.

MWDs are also found in CVs, and are called magnetic Cataclysmic Variables (MCVs). They comprise $\sim 20-25 \%$ of all known CVs. Zeeman and cyclotron spectroscopy of MCVs have revealed the presence of fields in the range $\sim 7-230$ MG. Complex field geometries have been inferred in the high field MCVs (the polars) whilst magnetic field strength and structure in the lower field group (intermediate polars, IPs) are much harder to establish.

To date there are about $\sim 250$ MWDs with well determined fields (as reported in the Table 1 of the review paper by Ferrario, de Martino \& Gänsicke, 2015) and over $\sim 600$ if we also count objects with no or uncertain field determination (see Kepler et al. 2013, 2015). The number of identified IPs has now increased to $\sim 60$ systems (see Table 3 of the review paper by Ferrario, de Martino \& Gänsicke, 2015 and updated results in Bernardini et al. (2015). The other $\sim 600$ 
candidates still awaiting confirmation through X-ray follow-ups with sensitive facilities such as XMM-Newton and NuSTAR (see http://asd.gsfc.nasa.gov/Koji.Mukai/iphome/iphome.html).

Enormous progress has been made on observing stellar magnetism in stars from the main sequence through to compact objects. Recent data have thrown into sharper relief the vexed question of the origin of stellar magnetic fields, which remains one of the main unanswered questions in astrophysics.

HMWDs have been long suspected to be the result of stellar mergers. However, the nature of the coalescing stars and the precise mechanism that produces the magnetic field are still unknown. García-Berro et al $(2012,2013)$ show that the hot, convective, differentially rotating corona present in the outer layers of the remnant of the merger of two degenerate cores is able to produce magnetic fields of the required strength that do not decay for long timescales. They also show, using a stateof-the-art Monte Carlo simulator, that the expected number of HMWDs produced in this way is consistent with that found in the solar neighborhood. The impact of mergers in the mass distribution of WDs has been discussed by Isern et al. (2013). Their toy model indicates that effectively the interaction of stars in close enough binary systems can introduce important changes in the expected mass distribution of WDs resulting from the evolution of single stars. Besides the existence of $\mathrm{He}$ WDs, the merging of such stars can produce a pronounced bump around 0.7-0.8 $\mathrm{M}_{\odot}$. The exact location and shape of this bump depends on the details of the merging process. The merger of $\mathrm{CO}$ and $\mathrm{He}$ and of two CO WDs only introduces small variations in the mass distribution. In the case of hot WDs they found a bump at $\sim 1 \mathrm{M}_{\odot}$ caused by the dependence of the cooling rate on the mass of the WD. This bump is absent in the mass function obtained from the SDSS catalogues but there is a hint of it in the mass function of the local sample.

Ferrario, Melatos \& Zrake (2015) review recent work in this area of research. In particular, they look at the fossil field hypothesis which links magnetism in compact stars to magnetism in main sequence and pre-main sequence stars and they consider why its feasibility has now been questioned particularly in the context of highly magnetic white dwarfs. They also review the fossil versus dynamo debate in the context of neutron stars and the roles played by key physical processes such as buoyancy, helicity, and superfluid turbulence, in the generation and stability of neutron star fields. Independent information on the internal magnetic field of neutron stars will come from future gravitational wave detections. The Laser Interferometer Gravitational Wave Observatory (LIGO) have already constrained the Crab pulsar gravitational wave luminosity to be $\lesssim 2 \%$ of the observed spin-down luminosity, thus placing a limit of $\lesssim 10^{16} \mathrm{G}$ on the internal field. Therefore we are witnessing the dawn of a new era of exciting discoveries in compact star magnetism driven by the opening of a new, non-electromagnetic observational window.

It is interesting to mention the review by Beskin et al. (2016a) about the fundamental role that strong magnetic fields play in the universe.

However, we can say that CVs form a broad stellar family of highly variable and dynamical members. When it comes to explaining particulars about, e.g., the detailed interaction between the transferred matter and the WD's atmosphere; irregularities within regular photometric behaviour; turbulent transport in the disk; or the final fate of these objects, more is missing than what is known, rendering their study ever more challenging. At least, CVs are natural multi-wavelength laboratories offering us the possibility of studying in detail the behaviour of plasma and radiation under extreme physical conditions (e.g. review by Giovannelli \& Sabau-Graziati, 2015a). The 
understanding of stellar evolution, electromagnetism and polarization, mass and radiation transfer or 3-D geometrical effects, in a broad spectral range from hard X-rays to radio, is mandatory for improving the knowledge of the nature of CVs.

Variability, from milliseconds to hundreds of years, follows from different physical processes taking place in these systems and can be studied by means of several astronomical techniques. As our skills in developing further these techniques grow our understanding of the CVs insights also grows; and the more we learn about CVs the further techniques and theory develop. On the other hand, it is well known that conclusions obtained in the field of CVs have been extrapolated, upwards or downwards in scale, to other fields such as AGNs or LMXRBs, and vice versa. From such exchanges of information and results astrophysical research in general always benefits. Rapid oscillations in CVs are particularly interesting. As reviewed by Warner (2004), the rich phenomenology of dwarf nova oscillations (DNOs) and quasi-periodic oscillations (QPOs) observed in CVs favour the interpretation that these rapid brightness modulations (3 to 11,000 s timescales) are magnetic in nature - magnetically channelled accretion from the inner accretion disk for DNOs and possible magnetically excited traveling waves in the disk for QPOs. There is increasing evidence for the magnetic aspects, which extend to lower fields the well-known properties of strong field (PCVs) and intermediate strength field (IPCVs) CVs. The result is that almost all CVs show the presence of magnetic fields on their WD primaries, although for many the intrinsic field may be locally enhanced by the accretion process itself. There are many behaviour that parallel the QPOs seen in $\mathrm{X}$-ray binaries, with high- and low-frequency X-ray QPOs resembling, respectively, the DNOs and QPOs in CVs. Other papers about rapid oscillations in CVs are those by Warner \& Woudt (2005) and Pretorius, Warner \& Woudt (2006).

The current estimate of the space density of CVs is of $\sim 3 \times 10^{-6} \mathrm{pc}^{-3}$ (Warner, 2001). This may be a significant underestimate of CVs space density, as discussed by Patterson (1984). Although densities from the most comprehensive optical Palomar-Green survey raises the estimate at $(3-6) \times 10^{-6} \mathrm{pc}^{-3}$, X-ray All-Sky surveys give densities of $\sim 1 \times 10^{-5} \mathrm{pc}^{-3}$ for detected systems of low $\dot{M}$ in hard X-rays (Patterson, 1998). Then from observational point of view, it is necessary an intensive search for the faint $\mathrm{CV}$ s predicted by population synthesis with orbital periods at $\sim 80-100 \mathrm{~min}$ that have passed through the orbital period minimum at $\sim 78 \mathrm{~min}$ and have increasing orbital periods. This research must be done among the low $\dot{\mathrm{M}}$ systems detected by X-ray surveys. Thanks to its high sensitivity, INTEGRAL is very useful for this purpose. Up to now, it discovered several new faint CVs, with $\mathrm{P}_{\text {orb }}>3 \mathrm{hr}$, and only one with $\mathrm{P}_{\text {orb }}<3 \mathrm{hr}$ (e.g. Šimon et al., 2006; Hudec et al., 2008). High speed photometry of faint CVs have shown that: i) 1 of 10, TV Crv has $\mathrm{P}_{\text {orb }}=1.509 \mathrm{hr}$ (Woudt \& Warner, 2003); ii) 5 of 13 have $\mathrm{P}_{\text {orb }}<2 \mathrm{hr}$ (Woudt, Warner \& Pretorius, 2004); iii) 1 (CAL 86) of 12 has $\mathrm{P}_{\text {orb }}=1.587 \mathrm{hr}$ (Woudt, Warner \& Spark, 2005); iv) 3 of 11 have $\mathrm{P}_{\text {orb }}>3 \mathrm{hr}$ (Witham et al., 2007).

For reviews about CVs see the fundamental papers by Robinson (1976), Patterson (1984, 1994), Hack \& la Dous (1993), and the books of Warner (1995a) and Hellier (2001). More recent reviews are those by Connon Smith (2007), Giovannelli (2008), Giovannelli \& Sabau-Graziati (2012c, 2015a). The long review The Impact of Space Experiments on our Knowledge of the Physics of the Universe by Giovannelli \& Sabau-Graziati (2004) contains also a part devoted to CVs. 


\subsection{Magnetic field intensity measures}

It is convenient to remind how the magnetic field intensity can be measured. A detailed discussion about the field determination in isolated magnetic WDs and in WDs in binary systems is reported in the review paper by Ferrario, de Martino \& Gänsicke (2015). Direct measurements of the WD magnetic field strength in the high field magnetic CVs, the polars, can be obtained either (i) through Zeeman splitting of the photospheric hydrogen absorptions lines when these systems enter low accretion states or (ii) through the modeling of cyclotron emission features that characterizes the optical to IR spectra during intermediate and high accretion states (see Wickramasinghe \& Ferrario, 2000) or (iii) via the study of Zeeman features arising from the halo of matter surrounding the accretion shock.

Figure 4 shows the distribution of B for MCVs and MWDs (adopted from Ferrario, de Martino \& Gänsicke, 2015).

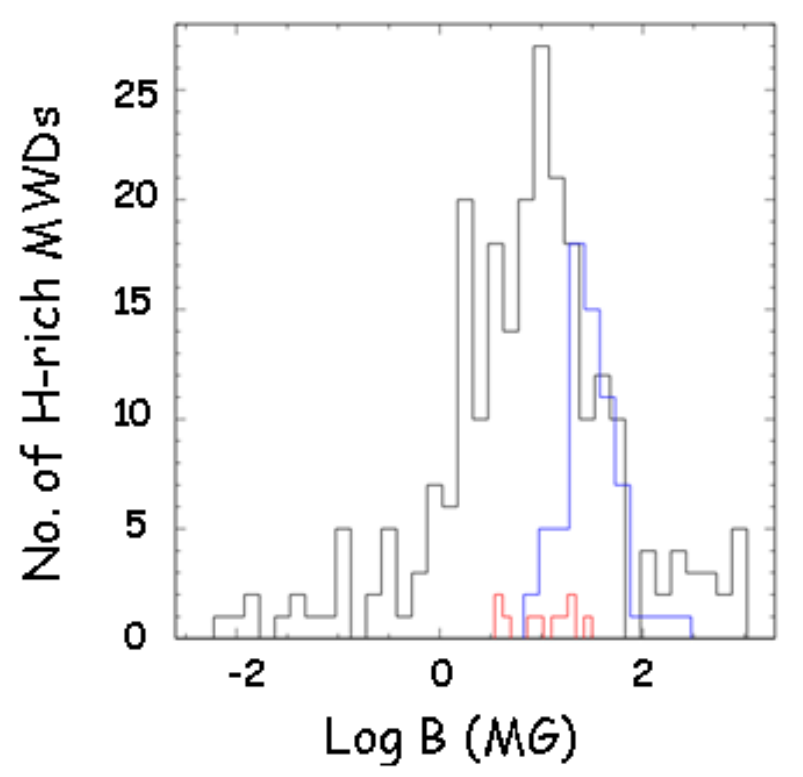

Figure 4: Distribution of magnetic field strength in polars (blue line), and IPs (red line) compared to that of single magnetic WDs (black line) (adapted from Ferrario, de Martino \& Gänsicke, 2015).

In MCVs there is an interesting relationship between the magnetic field strength and orbital period of the systems, as reported in Fig. 5 where the polars and IPs are separated by the blue line that marks the synchronization between orbital and spin periods of the cataclysmic systems (after Ferrario, de Martino \& Gänsicke, 2015).

Taking into account the average values of magnetic field intensity and orbital periods for polars and IPs, and the minimum and maximum value for both parameters ( $\mathrm{B}$ and $\mathrm{P}_{\mathrm{orb}}$ ), it is possible to construct a very interesting plot (Fig. 6) that shows the evident continuity between the two classes of MCVs. Such a continuity has been noted by Schmidtobreick \& Tappert $(2014,2015)$ : CVs evolution is driven by angular momentum loss; as consequence $\mathrm{P}_{\text {orb }}$ decreases. All long $\mathrm{P}_{\text {orb }} \mathrm{CVs}$ cross SW Sex regime before entering in the "period gap". Therefore SW Sex phenomenon is an evolutionary stage in the life of CVs (e.g. Rodriguez-Gil, 2003). 


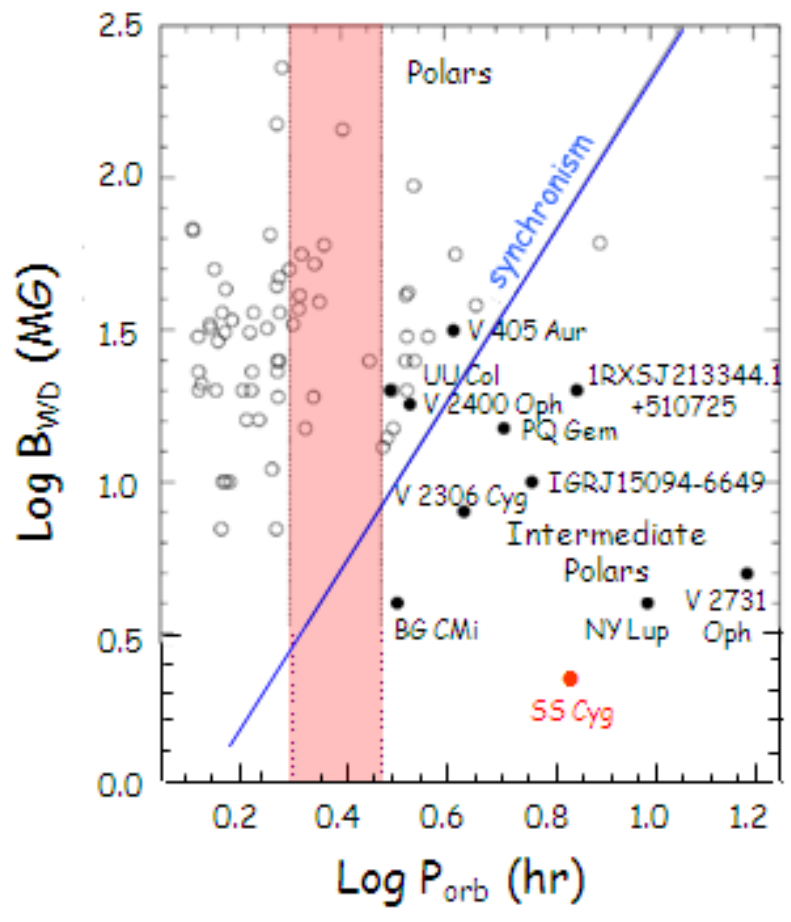

Figure 5: Magnetic field strength versus orbital period in MCVs. Polars and IPs are separated by the blue line that marks the border between systems with orbital period synchronized with the spin period (polars) and those without synchronization (IPs). Light red rectangle marks the so-called "period gap" (after Ferrario, de Martino \& Gänsicke, 2015).

An interesting indirect method for evaluating the magnetic field intensity in MCVs has been discussed by Giovannelli \& Sabau-Graziati (2012a) in the case of SS Cyg whose nature (non magnetic

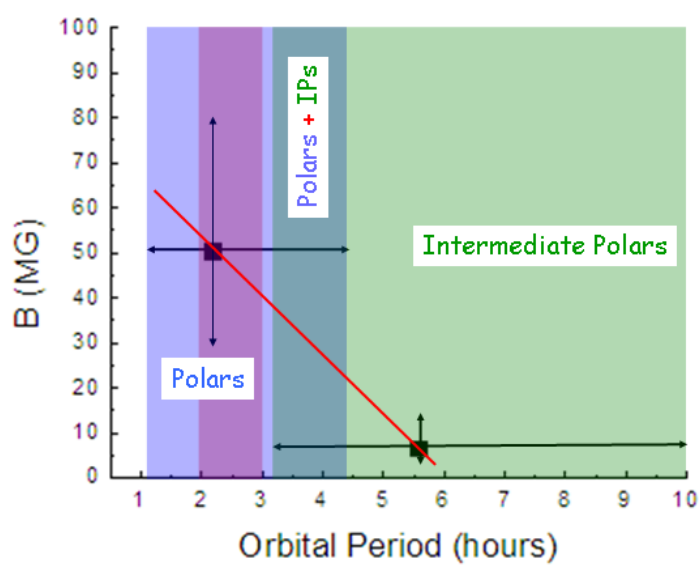

Figure 6: Magnetic field intensity versus orbital period for MCVs. Polars and IPs are contained in the light blue and light green rectangles, respectively. Violet rectangle indicates the so-called "period gap". Cyan-50 rectangle represents the intersection between the Polars and IPs. 
or IP) is largely disputed. From the fluxes of UV emission lines of SS Cyg, placed at distance d $=166 \pm 7$ pc (Harrison et al., 1999), Giovannelli \& Sabau-Graziati (2012a) - by using the IUE measurements obtained by Gaudenzi et al. (1986) - derived the luminosity of C II and C IV: $\mathrm{L}_{\mathrm{CII}} \simeq 7.8 \times 10^{30} \mathrm{erg} \mathrm{s}^{-1}$ and $\mathrm{L}_{\mathrm{CIV}} \simeq 6.2 \times 10^{31} \mathrm{erg} \mathrm{s}^{-1}$. Using these values of luminosity and the extrapolation of the line best fitting the emission line luminosity of C II and C IV versus B (Howell et al., 1999), the magnetic field intensity of SS Cyg is $\mathrm{B}_{\mathrm{CII}}=2.0_{-0.4}^{+0.5} \mathrm{MG}$, and $\mathrm{B}_{\mathrm{CIV}}=1.1_{-0.6}^{+0.3} \mathrm{MG}$, as shown in Fig. 7, left and right panels, respectively.
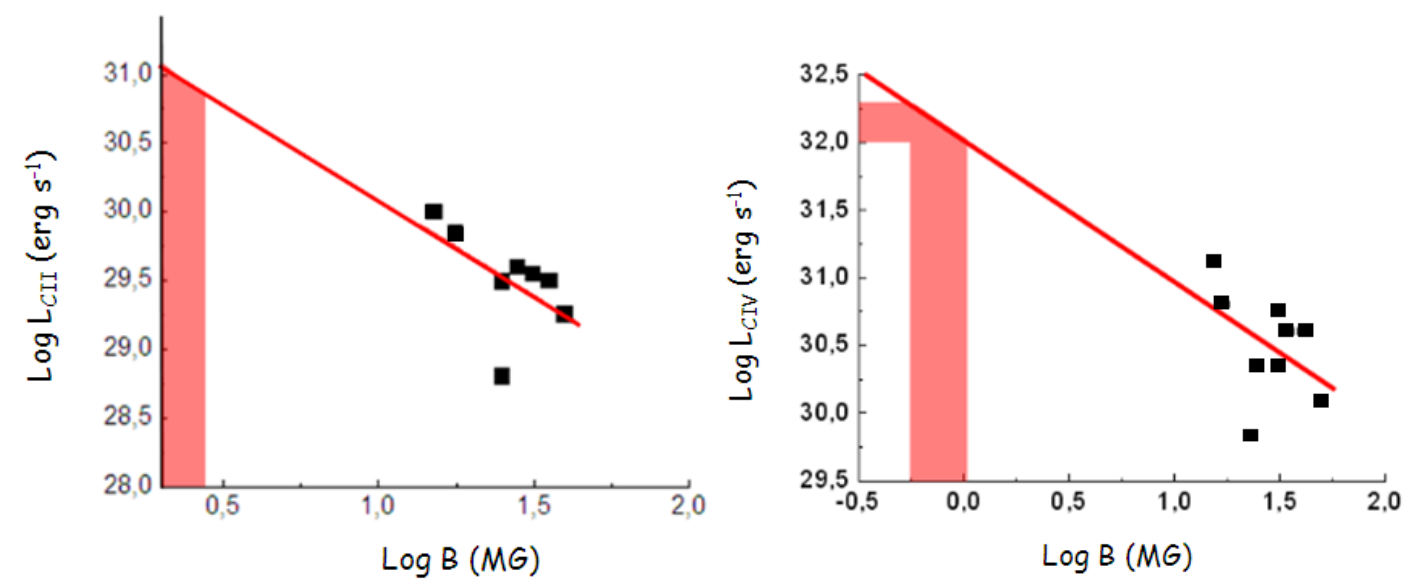

Figure 7: Observed emission fluxes converted to luminosity for magnetic CVs (after Howell et al., 1999) are indicated with black square. Left panel: C II luminosity versus B; right panel: C IV luminosity versus B. SS Cyg positions are indicated with red areas (Adopted from Giovannelli \& Sabau-Graziati, 2012a).

Then a reasonable value of the white dwarf magnetic field in SS Cyg is $\mathrm{B}=1.6 \pm 0.7 \mathrm{MG}$ This value is in complete agreement with the evaluation made by Fabbiano et al. (1981) $(\mathrm{B} \leq 1.9$ MG) by using simultaneous X-ray, UV, and optical data.

Many other circumstantial proofs in favor of the IP nature of SS Cyg have been discussed by Giovannelli \& Sabau-Graziati (2012a). One of the most important proof is coming from the paper by Körding et al. (2008). They detected a radio jet from SS Cyg. The hardness intensity diagram shows an analogy between the XRBs (the BH GX 339-4, and the NS Aql X-1) and SS Cyg. Moreover there is a radio flare simultaneous with the optical outburst of SS Cyg. During the 1.1-mJy "flare" they found upper limits for the linear polarization and circular polarization of $3.2 \pm 2.7 \%$ and $-3.2 \pm 2.7 \%$, respectively.

It is hard to explain these results without invoking the presence of a magnetic field ( $\mathrm{B} \approx 2$ MG) in the white dwarf of SS Cyg system.

INTEGRAL/IBIS and SWIFT/XRT observations have shown that a conspicuous number of CVs have a strong hard X-ray emission (Landi et al., 2009; Scaringi et al., 2010). In their published sample of $23 \mathrm{CVs}, 22$ are classified as magnetic IPs and only one (SS Cyg) as NMCV, meanwhile all its characteristics are practically equal to those of the other 22 objects. This is a strong circumstantial proof in favor of the magnetic nature of SS Cyg. The experimental evidence that SS Cyg emits in the hard X-ray energy range is, in our opinion, the conclusive evidence about its magnetic nature. 
Moreover, a simple question arises: "why all the CVs detected by the INTEGRAL observatory are magnetic (IPs) and SS Cyg non-magnetic? If so, why the same INTEGRAL observatory did not detect any other DN?".

The hard X-ray emission detected in those CVs is possible only if a sufficiently high magnetic field is present in those systems. Only accretion onto magnetic poles justify the hard X-ray emission in quiescence.

Probably, the "mistake" about the nature of SS Cyg born after the publication of a paper in Nature by Bath \& van Paradijs (1983) where SS Cyg was classified as DN on the basis of optical behaviour, typical of DNe. This paper originated a bandwagon effect in the literature (see Michael Friedjung's comment in the first historical Frascati Workshop 1984: Giovannelli, 1985) that "obliged" almost all the subsequent authors to start the papers saying that SS Cyg is a DN (NMCVs), without paying attention to other possibilities well documented in the so-called second class literature.

Therefore, our suggestion is to reconsider the problem about the nature of SS Cyg without any a priori bias.

Our opinion is that a more appropriate investigation of the class of the so-called IPCVs is necessary.

However, all the discussion about CVs would teach a lesson: it is mandatory to observe CVs for long time and possibly with simultaneous observations across the electromagnetic spectrum, from radio to gamma rays in order to follow at least a whole period of the binary system between two successive outbursts. This is, of course, possible only for systems like dwarf novae where the almost periodical outbursts occur in time scales of weeks-months. Networks of robotic telescopes can help in this matter (Giovannelli \& Sabau-Graziati, 2012b)

\section{Renewed Interest for Cataclysmic Variables}

Before the advent of ROSAT X-ray satellite, MCVs were relegated to a subsection of conferences about CVs that were mainly concentrated on NMCVs. The ROSAT satellite discovered many MCVs that even menaced to overthrow our understanding of the secular evolution of 'normal' CVs by appearing - apparently inexplicable - in the so-called 'period gap' in the orbital-distribution of CVs (e.g. Vrielmann \& Cropper, 2004). But in spite of this, CVs were not considered, in general, for many years as principal targets of high energy X-ray experiments.

At the beginning of the nineties of the last century, acceleration of particles by the rotating magnetic field of the WD in intermediate polars in the propeller regime - AE Aqr - detected by ground-based Cherenkov telescopes in the TeV passband (e.g. Meintjes et al. 1992), and TeV emission from the polar AM Her detected by ground-based Cherenkov telescopes (Bhat et al. 1991) - measurements never confirmed - were the main reasons of renewed interest for CVs in the high energy astrophysicists community.

Indeed, many measurements were later performed after the news about the possibility of VHE emission from AE Aqr. Chadwick et al. (1995) reported an excess of pulsed VHE $\gamma$ from AE Aqr on 1993 October 11, with the University of Durham VHE $\gamma$-ray telescope at Narrabri, Australia. The burst lasted $\sim 4200 \mathrm{~s}$ at phase $0.62-0.74$ of the $9.88 \mathrm{~h}$ orbital period. Lang et al. (1998) observed AE Aqr for a total of 68.7 hours over the epoch 1991-95 using the Whipple Observatory 
10-m $\gamma$-ray telescope. No evidence for any steady, pulsed or episodic $\mathrm{TeV}$ emission. Sidro et al. (2008) reported the observations of AE Aqr with the MAGIC telescope, performed in August 2005 during four consecutive nights within the context of a quasi-simultaneous multi-wavelength campaign covering the radio, optical, UV, and X-ray ranges. The analysis of these data revealed no evidence for any steady or pulsed $\gamma$-ray emission.

Meintjes, Oruru \& Odendaal (2012) discussed the multifrequency properties of AE Aqr within the framework of its secular evolution and a very effective magnetospheric propeller process. The very peculiar broad band emission from radio to possibly $\mathrm{TeV} \gamma$-rays is a manifestation of the propeller driven spin-down power of the white dwarf, which is $\mathrm{L}_{\text {prop }} \sim 10^{34} \mathrm{erg} \mathrm{s}^{-1}$. This may put $\mathrm{AE}$ Aqr in the class of rotation-powered pulsars, and provide an attractive framework to explain the earlier reports of the transient VHE-TeV $\gamma$-ray emission. It is remarkable to notice that AE Aqr lies just in the propeller area in the Lipunov's diagram reported in Fig. 1.

Aleksic et al. (2014) with the MAGIC experiment searched for steady $\gamma$-ray emission from AE Aqr during $12 \mathrm{~h}$ as part of a multi-wavelength campaign carried out between May and June 2012 covering the optical, $X$-ray, and $\gamma$-ray ranges. They did not find any significant $\gamma$-ray emission from AE Aqr in any of the searches performed.

The INTEGRAL observatory, until the beginning of 2007, had observed over 70 percent of the sky, with a total exposure time of 40 million seconds. Bird et al. (2007) published the third INTEGRAL catalogue of gamma-ray sources. It contains a total of 421 gamma-ray objects. Most have been identified as either binary stars in our Galaxy containing exotic objects such as black holes and neutron stars, or active galaxies, far away in space. But a puzzling quarter of sources remain unidentified so far. They could be either star systems enshrouded in dust and gas, or CVs. Integral observes in the gamma-ray band so it can see through the intervening material. It has demonstrated that it can discover sources obscured at other wavelengths. One surprise has been the efficiency with which INTEGRAL has detected just one minor subclass CVs, the so-called IPCVs. Initially astronomers were not sure that CVs would emit gamma rays. Indeed, INTEGRAL has already shown that only about one percent of them do. This fact overbearingly renewed the interest for $\mathrm{CV}$ s, apparently fallen into disgrace in favour of binary systems containing either neutron stars or black holes. The fourth IBIS/ISGRI catalog reports 331 additional sources when compared to the third catalog. Of these, 120 are associated with extragalactic sources, while only 25 are associated with known Galactic sources, and the remainder are so far unidentied (Bird et al. 2010). CVs constitute $\sim 5 \%$ of the total sources.

Moreover, since the CVs measured by the INTEGRAL observatory are magnetic in nature, the interest for such class of objects has been addressed to evolutionary problems.

The long-standing fundamental predictions of evolution theory are finally being tested observationally. All facets of the accretion process in CVs, including variability, disk winds and jets, are universal with accreting WDs, neutron stars, and black holes (Knigge, 2010, 2011). Knigge, Baraffe \& Patterson (2011) extensively discussed the reconstruction of the complete evolutionary path followed by CVs, based on the observed mass-radius relationship of their donor stars, following Knigge (2006) that discussed the observational and theoretical constraints on the global properties of secondary stars in CVs using the semi-empirical CV donor sequence, and concluded that most CVs follow a unique evolutionary track.

In the standard model of CV evolution, angular-momentum-loss (AML) below the period 
gap are assumed to be driven solely by gravitational radiation (GR), while AMLs above the gap are usually described by a magnetic bracking (MB) (Rappaport, Verbunt \& Joss (1983). Knigge, Baraffe \& Patterson (2011) with their revised model, found the optimal scale factors $\mathrm{f}_{\mathrm{GR}}=2.47$ below the gap and $\mathrm{f}_{\mathrm{MB}}=0.66$ above, whilst the standard model gives $\mathrm{f}_{\mathrm{GR}}=\mathrm{f}_{\mathrm{MB}}=1$. This revised model describes the mass-radius data much better than the standard model.

The sub-class of CVs, named Classical Novae $(\mathrm{CNe})$, which are the third more powerful stellar explosions in a galaxy, have been observed as close as a kpc and as far as galaxies in Fornax cluster. The time to report on the recent renaissance in studies on CNe thanks to observations with 8-10m class telescopes, high resolution spectroscopy, in synergy with observations from space carried out with Swift, XMM, Chandra, HST, and Spitzer, coupled with recent advances in the theory of the outburst, seems now in order. Moreover, the possible connection among some CV-types and SNe-Ia will definitively justify the renewed interest about CVs.

\section{Classical and Recurrent Novae}

Classical novae are expected to recur on timescales from 100,000 years to just a few decades. The most important physical parameters controlling this recurrence timescale are the WD mass, and the mass accretion rate from the secondary (e.g. Yaron et al. 2005). Once classical nova (CN) is recorded more than once, it can be designated as "recurrent" (RN). Since the WD and the binary system remain intact after an outburst, it is possible that classical novae may actually be the same as recurrent novae if observed over a long enough time period. While the interval between outbursts of recurrent novae range from 10 to 100 years, it has been estimated that the time interval for classical novae would range from about 30,000 years for a $1.3 \mathrm{M}_{\odot}$ WD to 100,000 years for a $0.6 \mathrm{M}_{\odot} \mathrm{WD}$. Given long enough - it is expected that all classical novae will be observed as recurrent novae.

The long term behaviour of classical old novae, and the optical behaviour of $\mathrm{CNe}$ in outburst were discussed by Bianchini (1990), and Seitter (1990), respectively. The books by Cassatella \& Viotti (1990) and by Bode \& Evans (2008) are very useful for studying the physics of classical novae.

Recurrent novae are a rare sub-class of CVs; WDs accreting material from a binary companion in which more than one classical nova-type outburst has been observed (see the book of Hellier, 2001 for a comprehensive review of CVs). Nova outbursts are suspected to be due to a thermonuclear runaway on the surface of the WD, which releases huge amounts of thermal energy once a critical pressure is reached at the base of the shell of accreted material.

One of the most interesting RNe is RS Ophiuchi (RS Oph). It is an amazingly prolific recurrent nova, with recorded outbursts in 1898, 1907, 1933, 1945, 1958, 1967, 1985 and 2006 (Schaefer 2010). The short time between outbursts ( 20 yrs) suggests that RS Oph hosts a massive WD accreting material at a significantly high rate.

In the latter paper Schaefer discussed not only RS Oph, but also the photometric histories of all known galactic RNe.

Classical and recurrent nova outbursts have been discussed by Bode (2011a,b) and Evans (2011). The proceedings of a conference about RS Oph and recurrent phenomenon can be very useful for details (Evans et al., 2008). General properties of quiescent novae have been discussed by Warner (2002). The very useful book of Bode \& Evans (2008) about classical novae examines 
thermonuclear processes, the evolution of nova systems, nova atmospheres and winds, the evolution of dust and molecules in novae, nova remnants, and observations of novae in other galaxies. It includes observations across the electromagnetic spectrum, from radio to gamma rays, and discusses some of the most important outstanding problems in classical nova research.

Of the $\sim 400$ known Galactic classical novae, only 10 of them are recurrent. Eight of them harbour evolved secondary stars, contrary to classical novae that contain main sequence stars (Darnley et al., 2011). They propose a new nova classification based on the evolutionary state of the secondary star, contrary the current schemes based on the properties of outbursts. Such classification contains three groups of novae: i) Main Sequence Nova (MS-Nova); ii) Sub-Giant Nova (SGNova); and iii) Red Giant branch Nova (RG-Nova).

An important not yet resolved problem is connected with the evolution and fate of Classical Novae. Patterson (2014) discussed this crucial problem. Classical novae rise from obscurity to shine among the brightest stars in the Galaxy. The story of how they return to quiescence is still only dimly known. Vast amounts of energy are loosed upon the WD and its companion, and the light curves of post-novae suggest that they take not a few years, but a few thousand years, to return to quiescence. In the meantime, the secondary may experience a lot of heating from the WD's radiation - enough to overwhelm its intrinsic nuclear luminosity. For this purpose he mentioned the case of BK Lyncis - the oldest old nova and a bell-wether for CVs evolution (Patterson et al., 2013). He discussed stellar physics behind this suggestion and proposed how it might be tested by time-series photometry in the months and years (and if possible, centuries) after outburst.

RNe play an important role in the studies of SN Ia progenitors (Surina, Bode \& Darnley, 2011). $\mathrm{RNe}$ are likely progenitors of Type-Ia supernovae.

On the contrary, Shafter et al. (2015) estimated that $\sim 4 \%$ of the nova eruptions seen in M31 over the past century are associated with RNe. A Monte Carlo analysis shows that the discovery efficiency for RNe may be as low as $10 \%$ that for novae in general, suggesting that as many as one in three nova eruptions observed in M31 arise from progenitor systems having recurrence times $\lesssim 100$ yr. For plausible system parameters, it appears unlikely that RNe can provide a significant channel for the production of Type-Ia supernovae.

Important works have been developed about extragalactic nova populations (Shafter et al., 2014). Nova rates have been measured for more than a dozen galaxies spanning a wide range of Hubble types. They found that the recurrent nova population in the LMC appears to be higher than that seen in M31 and the Galaxy.

In order to brave this important problem the use of archival data is the only way to answer the big question. Now, huge and comprehensive set of archival RN data go back to 1890.

\section{Progenitors of SN Ia}

It is well accepted by the community that Type-Ia SNe are the result of the explosion of a carbon-oxygen WD that grows to near Chandrasekhar's limit in a close binary system (Hoyle \& Fowler, 1960). But the debate is focussed around the different kinds of progenitors. Indeed, in the past, two families of progenitor models have been proposed. They differ in the mode of WD mass increase. The first family is the so-called single degenerate (SD) model (Whelan \& Iben, 1973), in which the WD accretes and burns hydrogen-rich material from the companion. The second family 
is the so-called double degenerate (DD) model, in which the merging of two WDs in a close binary triggers the explosion (Webbing, 1984; Iben \& Tutukov, 1984). The two scenarios produce different delay times for the birth of the binary system to explosion. Thus it is hopefully possible to discover the progenitors of Type-Ia SNe by studying their delay time distribution (DDT). The DDT can be determined empirically from the lag between the cosmic star formation rate and Type-Ia SN birthrate.

The energy released through runaway thermonuclear process ejects the majority of the unburnt hydrogen from the surface of the star in a shell of material moving at speeds of up to $1.5 \times 10^{3} \mathrm{~km}$ $\mathrm{s}^{-1}$. This produces a bright but short-lived burst of light - the nova.

Although Type-Ia supernovae appear to have similar origin to classical novae, there are key differences. The most important is that in a classical nova, the thermonuclear runaway occurs only on the surface of the star, allowing the WD and the binary system to remain intact (e.g. Townsley \& Bildsten, 2005). In a Type-Ia supernova, the thermonuclear runaway occurs within WD itself, completely disrupting the progenitor. This is reflected in the amount of energy released in the explosions, with classical novae releasing $\sim 10^{44} \mathrm{erg}$, and Type-Ia supernovae $\sim 10^{51} \mathrm{erg}$.

The possible progenitors of SN Ia are: i) Recurrent Novae; ii) Symbiotic stars; iii) Super-soft sources; iv) Double WD Binaries; and v) WDs accreting material from red-giant companions.

i) Recurrent Novae are just a subset of ordinary novae that happen to go off more than once per century.

As such, they are binary systems with matter flowing off a companion star onto a WD, accumulating on its surface until the pressure gets high enough to trigger a thermonuclear runaway that is the nova.

Only 10 RNe are known in our Milky Way galaxy, including: U Sco $(1863,1907,1917,1936$, 1945, 1969, 1979, 1987, 1999); Т Pyx (1890, 1902, 1920, 1944, 1967); T CrB (1866, 1946); RS Oph $(1898,1907,1933,1945,1958,1967,1985,2006)$.

To recur with $\tau_{\text {rec }}<100$ years, RNe must have: high WD mass $\left(1.2 \mathrm{M}_{\odot}<\mathrm{M}_{\mathrm{WD}}<\mathrm{M}_{\text {Chandra }}\right)$, and high accretion rate $\left(\dot{\mathrm{M}} \sim 10^{-7} \mathrm{M}_{\odot} \mathrm{yr}^{-1}\right)$. SN Ia occurs if: i) the mass ejected for each eruption is less than the mass accreted onto the WD $\left(\mathrm{M}_{\text {ejected }}<\dot{\mathrm{M}} \tau_{\text {rec }}\right)$; ii) the rate of death RNe must be enough to produce the $S N$ Ia rate $\left(R_{R N d e a t h}=R_{S N I a}\right)$, being $R_{R N d e a t h}=N_{R N} \times\left(0.2 M_{\odot} \dot{M}\right)$.

In order to solve the problems we need to know $\tau_{\text {rec }}$ (recurrence time scale) from archive plates, $\mathrm{N}_{\mathrm{RN}}$ (number of RNe in the Milky Way) from archive plates and AAVSO, $\dot{\mathrm{M}}$ (mass accretion rate onto WD) from the average in the last century, $\mathrm{M}_{\mathrm{ejected}}$ (mass ejected in eruption) from pre-eruption eclipse timing.

Some results have been obtained for becoming optimists in solving the problem of SN Ia production. Indeed Schaefer (2011) obtained for CI Aql and U Sco $\mathrm{M}_{\text {ejected }}<<\dot{\mathrm{M}} \tau_{\text {rec }}$ ).

Thus, WDs are gaining mass and the latter RNe will collapse as SN Ia. Moreover, for the Milky Way, M31, and LMC $\mathrm{R}_{\mathrm{RNdeath}} \sim \mathrm{N}_{\mathrm{RN}}$. Then there are enough $\mathrm{RNe}$ to supply the Type-Ia $\mathrm{SN}$ events.

ii) Symbiotic Stars contain WDs efficiently accreting material from the secondary star. In most cases they steadily burn $\mathrm{H}$-rich material allowing them to grow in mass. Some of these systems can produce high mass WDs. In symbiotic RNe (SyRNe) the WD mass is already very close to Chandrasekhar's limit. For instance in V $407 \mathrm{Cyg}$ a very massive WD is accreting material at a rate of $\sim 10^{-7} \mathrm{M}_{\odot} \mathrm{yr}^{-1}$ from a Mira-type companion (Mikołajewska, 2011). 
iii) Super-soft Sources are probably WDs that accrete material and burn hydrogen. Voss $\&$ Nelemans (2008) discovered an object at the position of the Type-Ia SN2007on in the elliptical galaxy NGC1404 on pre-supernova archival X-ray images. This result favours the accretion model (SD) for this supernova, although the host galaxy is older than the age at which the explosions are predicted in SD models. However, the DD model cannot be ruled out by this event because a hot accretion disc is probably the intermediate configuration of the system, between first WD-WD Roche-lobe contact and explosion (Yoon, Podsiadlowski \& Rosswog, 2007).

Greggio, Renzini \& Daddi (2008) starting from the fact that Type-Ia SN events occur over an extended period of time, following a distribution of delay times (DDT), discussed theoretical DDT functions that accommodate both 'prompt" and 'tardy' SN events derived by empirically-based DDT functions. Moreover such theoretical DDT functions can account for all available observational constraints. The result is that SD/DD mix of SNIa's is predicted to vary in a systematic fashion as function of cosmic time (redshift).

iv) Double WDs Binaries are systems containing two WDs that can merge and giving rise to SN explosion. Yoon, Podsiadlowski \& Rosswog (2007) explored the evolution of the merger of two carbon-oxygen (CO) WDs. Their results imply that at least some products of double CO WDs merger may be considered good candidates for the progenitors of Type-Ia SNe. Brown et al. (2011) and Kilic et al. (2011) studied a complete colour-selected sample of double-degenerate binary systems containing extremely low mass $(E L M)\left(\leq 0.25 \mathrm{M}_{\odot}\right)$ WDs. Milky Way disc ELM WDs have a merger rate of $\approx 4 \times 10^{-5} \mathrm{yr}^{-1}$ due to gravitational wave radiation. The ELM WD systems that undergo stable mass transfer can account for about $3 \%$ of AM CVn stars. The most important fact is that the ELM WD systems that may detonate merge at a rate comparable to the estimate rate of underluminous SNe. These $\mathrm{SNe}$ are rare explosions estimated to produce only $\sim 0.2 \mathrm{M}_{\odot}$ worth of ejecta. At least $25 \%$ of ELM WD sample belong to the old tick disc and halo components of our Galaxy. Thus, if merging ELM WD systems are the progenitors of underluminous SNe, transient surveys must find them in both elliptical and spiral galaxies.

v) WDs accreting material from red-giant companions. Observations carried out by Patat et al. (2008) with VLT-UVES allowed to detect circumstellar material in a normal Type-Ia SN. The expansion velocities, densities and dimensions of the circumstellar envelope indicate that this material was ejected from the system prior to the explosion. The relatively low expansion velocities favour a progenitor system where a WD accretes material from a companion star, which is in the red-giant phase at the time of explosion.

Bianco et al. (2011) searched for a signature of a non-degenerate companion in three years of Supernova Legacy Survey data. They found that a contribution from WD/red-giant binary system to Type-Ia SN explosions greater than $10 \%$ at $2 \sigma$, and than $20 \%$ at $3 \sigma$ level is ruled out.

Type-Ia SNe are used as primary distance indicators in cosmology (e.g. Phillips, 2005). Phillips (2011) reviewed the near-infrared (NIR) of Type-Ia SNe concluding that such SNe are essentially perfect standard candles in the NIR, displaying only a slight dependence of peak luminosity on decline rate and colour. Lira (1995) first noted that B-V evolution during the period from 30 to 90 days after $\mathrm{V}$ maximum is remarkably similar for all SN Ia events, regardless of light-curve shape. This fact was used by Phillips et al. (1999) to calibrate the dependence of the $B_{\max }-V_{\max }$ and $\mathrm{V}_{\max }-\mathrm{I}_{\max }$ colours on the light curve parameter $\Delta \mathrm{m}_{15}$ (B) which can, in turn, be used to sep- 
arately evaluate the host galaxy extinction. Using these methods for eliminating the effect of the reddening, they reanalyzed the functional form of the decline rate versus luminosity relationship and gave a value of the Hubble constant of $\mathrm{H}_{0}=63.3 \pm 2.2 \pm 3.3 \mathrm{~km} \mathrm{~s}^{-1} \mathrm{Mpc}^{-1}$.

The use of Type-Ia SNe is also fundamental for determining some cosmological constraints, such as $\Omega_{\mathrm{M}}$ and $\Omega_{\Lambda}$ that fit a $\Lambda \mathrm{CDM}$ models with values of $0.211 \pm 0.034$ (stat) \pm 0.069 (sys) using a set of 252 high-redshift SNe (Guy et al., 2010) and $0.713_{-0.029}^{+0.027}$ (stat) ${ }_{-0.039}^{+0.036}$ (sys) using a set of low-redshift nearby-Hubble-flow SNe (Kowalski et al., 2008), respectively.

In order to explore the difficult topic of the expansion of the Universe it is necessary to know the evolution of metallicity in old Universe that changes the Hubble Diagram shape. The proposed space observatory Super Nova Acceleration Probe (SNAP) is designed to measure the expansion of the Universe and to determine the nature of the mysterious Dark Energy that is accelerating this expansion (Aldering, 2005). SNAP is being proposed as part of the Joint Dark Energy Mission (JDEM) (Stril, Cahn \& Linder, 2010), which is a cooperative venture between NASA and the U.S. Department of Energy. If selected it will be launched before 2020. SNAP cannot achieve its main goal without progenitor/evolution solution.

The research about the progenotors of SN Ia is of course one of the most important problems, since it is strictly connected with the evolution of CVs. For instance, Maguire et al. (2012) present an analysis of the maximum light, near-ultraviolet (NUV; $2900<\lambda<5500$ ) spectra of 32 lowredshift $(0.001<\mathrm{z}<0.08)$ SNe Ia, obtained with the Hubble Space Telescope (HST) using the Space Telescope Imaging Spectrograph. They combine this spectroscopic sample with high-quality gri light curves obtained with robotic telescopes to measure SN Ia photometric parameters, such as stretch (light-curve width), optical colour and brightness. They confirm and strengthen earlier conclusions regarding the complex behaviour of SNe Ia in the NUV spectral region, but suggest that the correlations found are more useful in putting tighter constraints on the progenitor systems of SNe Ia and how their progenitor channels may vary with host galaxy properties (e.g. metallicity: Kistler et al., 2013) rather than improving the use of SNe Ia as cosmological probes.

Darnley et al. (2014) discussed on the galactic nova progenitor population. They presented a selection of the work and rationale that led to the proposal of a new nova classification scheme based not on the outburst properties but on the nature of the quiescent system. They also outlined the results of a photometric survey of a sample of quiescent Galactic novae, showing that the evolutionary state of the secondary can be easily determined and leading to a number of predictions, including their relevance to extragalactic work and the proposed link to type-Ia SNe.

In order to solve the problem of determining the SN Ia progenitors, it is also important to look at the $\mathrm{RNe}$ that show many similarities to $\mathrm{CNe}$, but have had more than one recorded outburst. $\mathrm{RNe}$ play an important role as one of the suspected progenitor systems of Type-Ia SNe, which are used as primary distance indicators in cosmology. Thus, it is important to investigate the nature of their central binary systems to determine the relation between the parameters of the central system and the outburst type, and finally ascertain the population of novae that might be available to give rise to the progenitors of Type-Ia SNe. Surina, Bode \& Darnley (2015) adopted a low outburst amplitude as a criterion that may help distinguish $\mathrm{RNe}$ from $\mathrm{CNe}$ and was therefore used to select targets for observations from ground-based observatories including the Liverpool Telescope and the Southern African Large Telescope as well as the full-sky space-based archive of the Solar Mass Ejection Imager (SMEI). They found that at least four objects currently classified as $\mathrm{CNe}$ are 
possibly RNe candidates based on their quiescent spectra. They also searched the SMEI archive for additional outbursts of bright $\mathrm{CNe}$ that might otherwise have been missed but did not find a conclusive example.

Another possible channel for triggering the explosion of SN Ia is that discussed by Chiosi et al. (2015). They explore the possibility that isolated CO-WDs with mass smaller than the Chandrasekhar limit may undergo nuclear runaway and SN explosion. If this channel could be confirmed it should be possible (i) to explain the star formation rate dependence of the SN Ia rate (e.g. Mannucci, Della Valle \& Panagia, 2006); (ii) to provide some clues to interpreting the observational data on the ejected mass distribution of type-Ia SNe showing a significant rate of non-Chandrasekhar-mass progenitors of mass as low as $0.8 \mathrm{M}_{\odot}$ (Scalzo, Ruiter \& Sim, 2014); and (iii) to account for the $\mathrm{SNe}$ exploding inside Planetary Nebulae in alternative to the core-degenerate scenario in which a WD merges with the hot core of an AGB star on a time interval $\leq 10^{8} \mathrm{yr}$ since the WD formation (see Tsebrenko \& Soker, 2015, for more details). With the models of Chiosi et al. (2015), a single CO-WD may reach the explosion stage soon after the formation if sufficiently massive $\left(>1.0 \mathrm{M}_{\odot}\right)$ and sufficiently rich in residual hydrogen $\left(\mathrm{X}_{\mathrm{H}} \simeq 10^{-19}-10^{-20}\right)$. The expected time delay after formation can be as low as about a few ten of thousand years.

Williams et al. $(2014,2016)$ report the results of a survey of M31 novae in quiescence. The derived catalog contains data for 38 spectroscopically confirmed novae from 2006 to 2012. They used Liverpool Telescope images of each nova during eruption to define an accurate position for each system. These positions were then matched to archival Hubble Space Telescope (HST) images and they performed photometry on any resolved objects that were coincident with the eruption positions. This in order to facilitate a search for their progenitor systems within archival Hubble Space Telescope (HST) data, with the aim of detecting systems with red giant secondaries (RG-novae) or luminous accretion disks. They found an elevated proportion of nova systems with evolved secondaries that may imply the presence of a much larger population of recurrent novae than previously thought. This would have considerable impact, particularly with regards to their potential as TypeIa SN progenitors. Their results also imply that RG-novae in M31 are more likely to be associated with the M31 disk population than the bulge, indeed the results are consistent with all RG-novae residing in the disk. If this result is confirmed in other galaxies, it suggests any Type-Ia SNe that originate from RG-nova systems are more likely to be associated with younger populations, and may be rare in old stellar populations, such as early-type galaxies.

An important paper by Churazov et al. (2014) reports the first ever detection of ${ }^{56} \mathrm{Co}$ lines at 847 and $1237 \mathrm{keV}$ and a continuum in the $200-400 \mathrm{keV}$ band from the Type-Ia SN2014J in M82 with INTEGRAL observatory. The data were taken between 50th and 100th day since the SN2014J outburst. The line fluxes suggest that $0.62 \pm 0.13 \mathrm{M}_{\odot}$ of radioactive ${ }^{56} \mathrm{Ni}$ were synthesized during the explosion. Line broadening gives a characteristic ejecta expansion velocity $\mathrm{V}_{\mathrm{e}} \sim 2100 \pm 500$ $\mathrm{km} \mathrm{s}^{-1}$. The flux at lower energies (200-400 keV) is consistent with the three-photon positronium annihilation, Compton downscattering and absorption in the $\sim 1.4 \mathrm{M}_{\odot}$ ejecta composed from equal fractions of iron-group and intermediate-mass elements and a kinetic energy $\mathrm{E}_{\mathrm{k}} \sim 1.4 \times 10^{51}$ erg. All these parameters are in broad agreement with a "canonical" model of an explosion of a Chandrasekhar-mass WD, providing an unambiguous proof of the nature of Type-Ia SNe as a thermonuclear explosion of a solar mass compact object. Late optical spectra (day 136 after the explosion) show rather symmetric Co and Fe line profiles, suggesting that, unless the viewing an- 
gle is special, the distribution of radioactive elements is symmetric in the ejecta (Churazov et al., 2015).

For comments and prospects about Type-Ia SN science in the decade 2010-2020 see the paper by Howell et al. (2009).

\section{Some Open Questions}

Several fundamental questions concerning CVs still remain waiting for a proper answer. Here we will present briefly only some of them.

One of them is the lack of a coherent classification, especially for NLs. On the other hand, in gross features and in most respects, DN and NLs, as well as quiescent novae, are almost indistinguishable, although, in addition to their different outbursts' behaviour, there appear to be some further minor differences which are not yet understood (see Hack \& la Dous 1993). The question arises of whether the outburst behaviour, the current basis of almost all classification is really a suitable criterion for sorting CVs in physically related groups. There are also too many exceptions, either systems that do not fit in any particular group or that can be included in several of them, to be able to render the observational behaviour, at least as it is used at the present, suitable.

Could CVs be considered simply gravimagnetic rotators? This should be the most suitable approach for studying them from a physical point of view.

Studies of rotational equilibria of MCVs predict that IPCVs will evolve either into PCVs or into low field strength polars - presumably unobservable, and possibly EUV emitters - depending on their magnetic moments and orbital periods. Indeed, there are systems, like EX Hya-type, having magnetic moment similar to IPCVs above the 'period gap' and comparable to the weakest field AM Her-like systems.

Moreover, the detection of several SW Sex systems having orbital periods inside the so-called 'period gap' opens a new interesting problem about the continuity in the evolution of CVs.

The rare AM CVn stars have extremely short orbital periods, between 10 and 65 minutes, and their spectra show no evidence for hydrogen. They appear to be helium-rich versions of CVs. They are still waiting for a general model. They are probably binary systems of two white dwarfs, but even this is still controversial.

Despite all the work developed during the last decades, the problem of modeling accretion disks in CVs is by no means closed, especially in quiescence. Closely related is the problem of the cause of outbursts. We really do not know which of the present two families of models (Disk Instability Models or Secondary Instability Models) is responsible for the CVs outburst phenomenon, or in which system is each model valid, although Martinez-Pais et al. (1996) gave a contribution in solving this problem at least in the case of SS Cygni; they found some evidence for an increase of the mass transfer rate from the secondary star as the mechanism responsible for symmetric outbursts. Something similar can be said about the super-outburst phenomenon in SU UMa systems.

Gaudenzi et al. (1990), analyzing IUE spectra of SS Cygni, discussed about the outburst production as due to the destruction of the accretion disk. The matter slowly accretes onto the WD. Long and short outbursts correspond to total or partial destruction of the disk, respectively.

Alternatively, could nuclear burning be responsible of the production of outbursts in CVs? Indeed, nuclear burning onto white dwarf' surface was proposed by Mitrofanov $(1978,1980)$ as a 
mechanism suitable to generate X-rays in CVs. In spite of this shrewd suggestion, the community of theoreticians did not consider such a mechanism - certainly possible - worthy of taking up a part of their time. However, we believe that this alternative solution in explaining the generation of outbursts in CVs would deserve theoretician community's care. For instance, the white dwarf surface interested in the accretion in the system SS Cygni has been evaluated as $24 \%$ of the total (Gaudenzi et al., 2002). There, nuclear burning could occur.

Accretional heating by periodic DN events increases substantially the surface temperature of the WD in CVs (Godon \& Sion, 2002). Then, the envelope thermal structure resulting from compression and irradiation should be a crucial component in understanding the envelope structure of a pre-nova WD.

Another problem still open is connected with the classification of $\mathrm{CVs}$ in three kinds, namely NMCVs, PCVs and IPCVs. This is, in our opinion, another convenient classification, although artificial, probably not necessary if CVs are studied as gravimagnetic rotators. In this way a smooth evolution of the systems could be responsible of the variations of the gravimagnetic parameters.

Are the IPCVs and PCVs smoothly connected via the SW Sex-like systems placed just in between? SW Sex systems have indeed orbital periods belong to the so-called 'period gap', and then their presence there sure cancel that gap.

Could some systems behave in different ways depending on their instantaneous physical conditions? For this reason they could apparently behave sometimes as PCVs and sometimes as NPCVs.

An example very clear is that of SS Cygni, usually classified as a non-magnetic dwarf nova. It has been detected by the INTEGRAL observatory in a region of the spectrum (up to $\sim 100$ $\mathrm{keV})$. This emission is very hard to be explained without the presence of polar caps in the WD of the system. Several proofs have been shown and discussed many times by Giovannelli's group in order to demonstrate the Intermediate Polar nature of it (e.g., Giovannelli, 1996, and references therein; Giovannelli \& Sabau-Graziati, 1998; 2012a); indeed, SS Cygni shows characteristics of a NMCV, as well as those of IP and sometimes even those of polars, although its position in the $\log \mathrm{P}_{\text {spin }}-\log \mathrm{P}_{\text {orb }}$ plane is very close to the line where IPs lie.

Important results are coming from the SPITZER space telescope with the detection of an excess (3-8) $\mu \mathrm{m}$ emission from MCVs, due to dust (Howell et al., 2006; Brinkworth et al., 2007). Gaudenzi et al. (2011) discussed about the reasons of the variable reddening in SS Cyg and demonstrated that this reddening is formed by two components: the first is interstellar in origin, and the second (intrinsic to the system itself) is variable and changes during the evolution of a quiescent phase. Moreover, an orbital modulation also exists. The physical and chemical parameters of the system are consistent with the possibility of formation of fullerenes.

The SPITZER space telescope detected the presence of fullerenes in a young planetary nebula (Cami et al., 2010). Fullerenes are the first bricks for the emergence of the life. Therefore, the possible presence of fullerenes in CVs opens a new line of investigation, foreboding of new interesting surprises.

Further information can be considered in order to better synthetize the open problems in the knowledge of CVs and related objects.

Sion (http://astronomy.villanova.edu/faculty/sion/CV/index.html) states that in the Galaxy we could expect $\approx 10^{6} \mathrm{CVs}$. One of the big questions that arises is: "can all of the observed CVs and the phenomena associated with them be understood in terms of a single unified picture?" Other 
questions relate to the relative probabilities that CVs will be observed at particular stages in their evolution, and how the observations of CVs at the current epoch can be used to determine their ultimate fate. To address these questions Nelson (2012) and Goliasch \& Nelson (2015) have undertaken a massive computational effort to theoretically simulate the evolution of most of the possible $\mathrm{CVs}$ that could be produced by nature. The temporal evolution of 56,000 nascent CVs was followed over an age of 10 billion years using the MESA stellar evolution code. According to Nelson, "This is the most ambitious analysis of the properties of an entire CV population that has ever been undertaken. The whole project required several core-years of CPU time."

While many of the results confirmed what had already been inferred about the properties of $\mathrm{CVs}$, there were a number of surprises including the identification of a number of previously unexplored evolutionary pathways. But, as expected, a sharp bifurcation was found between nascent CVs that evolved to produce double white-dwarf binaries (including ones containing helium and hybrid white dwarfs), and ones that continuously transferred mass over the lifetime of the universe. In addition, the predictions of the theoretical simulations were in good general agreement with the observations of CVs with reasonably well-measured properties.

What was surprising was the large number of short-period "ultracompact" binaries (AM CVn stars) that were produced and, especially, the enormous depletion of carbon relative to nitrogen and oxygen that is predicted at certain epochs for evolved systems. As Nelson points out, "It seems that nature has provided us with a unique way to identify CVs that descended from a highly evolved state based on their carbon abundances. There is already some observational evidence to suggest that there is a significant depletion of carbon in certain CVs. This could be a really critical test that will allow us to infer the lineage of some CVs and predict what their fate will be".

Szkody \& Gänsicke (2012) provided a list of unanswered problems and questions and references for seeking additional information. Indeed, while the general evolutionary picture and the characteristics of the types of CVs are known at some level, there are major unsolved questions which remain. These include:

1. What is the actual number density and distribution of CVs in the Galaxy?

2. What happens to CVs once they reach the period minimum?

3. What are the detailed physics occurring in the common envelope?

4. What is the correct physics to describe viscosity in accretion disks?

5. What is the correct angular momentum prescription below the gap (besides gravitational radiation) that can account for the observed period minimum spike and the exact period distribution?

6. What causes the period gap?

7. How do Polars form and why are no magnetic white dwarfs in wide binaries observed? Are LARPS (Low Accretion Rate Polars) the progenitors of polars? Is there a difference in the emergence of systems containing magnetic white dwarfs versus non-magnetic? 
8. What causes Polars, as well as the novalike disk systems with orbital periods between 3 and 4 hours, to cease mass transfer and enter low states? Are the associated mass transfer variations of the companion stars a general phenomenon among all CVs?

9. Can the white dwarfs in CVs grow in mass?

10. Do CVs contain exoplanets?

In order to answer to these not yet solved problems, a series of biennial Palermo Workshops about "The Golden Age of Cataclysmic Variables and Related Objects" has been organized since 2011. The refereed proceedings can be found in Giovannelli \& Sabau-Graziati (2012c, 2015c, 2017).

\section{Conclusions}

At the end of this review it appears evident that the most suitable approach for studying CVs from a physical point of view is to consider them as gravimagnetic rotators.

The detection of several SW Sex systems having orbital periods inside the so-called 'period gap' opens a new interesting problem about the continuity in the evolution of CVs. Are the IPCVs and PCVs smoothly connected via the SW Sex-like systems placed just in between?

In order to fully understand the emission properties and evolution of $\mathrm{CVs}$, the mass-transfer process needs to be clearly understood, especially magnetic mass transfer, as well as the properties of magnetic viscosity in the accretion disks around compact objects. Consequently, the investigation on the magnetic field intensities in WDs appears crucial in understanding the evolution of CVs systems, by which it is possible to generate classical novae (e.g., Isern et al., 1997) and type-Ia supernovae (e.g., Isern et al., 1993).

In those catastrophic processes the production of light and heavy elements, and then the knowledge of their abundances provides strong direct inputs for cosmological models and cosmic ray generation problems.

We want to conclude with a general warning, apparently underestimated, like remarked by Giovannelli \& Sabau-Graziati (2015b): if we have not experimental information about the cross sections of nuclear reactions occurring in the stars it is hard to describe the correct star evolution.

The LUNA (Laboratory for Underground Nuclear Astrophysics) is devoted to measure nuclear cross sections relevant in astrophysics and astroparticle physics. It is the most valuable experiment running underground in the Gran Sasso Laboratory of the INFN (e.g. review by Broggini et al., 2010). LUNA experiment provided the measures of the cross-sections of many nuclear reactions occurring in the stars for a better knowledge of stellar evolution (see Giovannelli \& Sabau-Graziati, 2015b, and references therein).

A general data base for Experimental Nuclear Reaction Data (EXFOR) can be found in: https://www-nds.iaea.org/exfor/exfor.htm.

Acknowledgments This research has made use of NASA's Astrophysics Data System. 


\section{References}

[1] Aldering, G.: 2005, NewAR 49, 346-353.

[2] Aleksic, J., Ansoldi, S., Antonelli, L.A., Antoranz, P., Babic, A. et al.: 2014, A\&A, 568, id. A109, 8 pp.

[3] Andronov, I.L., Chinarova, L.L., Han, W., Kim, Y., Yoon, J.-N.: 2008, A\&A, 486, 855.

[4] Ak, T., Bilir, S., Ak, S., Coşkunoğlu, K.B., Eker, Z.: 2010, New Astron. 15, 491.

[5] Araujo-Betancor, S., Gänsicke, B.T., Long, K.S.,; Beuermann, K., de Martino, D., et al.: 2005, ApJ, $622,589$.

[6] Bath, G.T., van Paradijs, J.: 1983, Nature, 305, 33.

[7] Bhat, C.L., Kaul, R.K., Rawat, H.S., Senecha, V.K., Rannot, R.C., et al.: 1991, ApJ, 369, 475.

[8] Beskin, V.S., Balogh, A., Falanga, M., Treumann, R.A.: 2016a, in The Strongest Magnetic Fields in the Universe, Beskin, V.S., Balogh, A., Falanga, M., Lyutikov, M., Mereghetti, S. et al. (Eds.), Space Science Series of ISSI, Vol 54, 3.

[9] Beskin, V.S., Balogh, A., Falanga, M., Lyutikov, M., Mereghetti, S. et al. (Eds.): 2016b, The Strongest Magnetic Fields in the Universe, Space Science Series of ISSI, Vol 54, Springer, pp. 579.

[10] Bernardini, F., de Martino, D., Mukai, K., Israel, G., Falanga, M. et al.: 2015, MNRAS 453, 3100.

[11] Beuermann, K.: 1998, in High Energy Astronomy and Astrophysics, P.C. Agrawal \& P.R. Visvanathan (eds.), India Univ. Press, p. 100.

[12] Bianchini, A.: 1990, in Physics of Classical Novae, A. Cassatella \& R. Viotti (eds.), Springer-Verlag, Lecture Notes in Physics, 369, 13.

[13] Bianco, F.B., Howell, D.A., Sullivan, M., Conley, A., Kasen, D., et al.: 2011, ApJ, 741, 20.

[14] Bird, A.J., Malizia, A., Bazzano, A., Barlow, E.J., Bassani, L., et al.: 2007, ApJS, 170, 175.

[15] Bird, A.J., Bazzano, A., Bassani, L., Capitanio, F., Fiocchi, M., et al.: 2010, ApJS, 186, 1.

[16] Bode, M.F.: 2011a, arXiv: 0911.5254v3.

[17] Bode, M.F.: 2011b, arXiv: 111.4941v1.

[18] Bode, M.F., Evans, A. (eds.): 2008, Classical Novae, Cambridge Astrophys. Ser., No. 43, Cambridge University Press.

[19] Boyd, D., Patterson, J., Allen, W., Bolt, G., Bonnardeau, M. et al.: 2014, in 33rd Annual Symposium on Telescope Science, Society for Astron. Sci., 33, 163-176.

[20] Brinkworth, C.S., Hoard, D.W., Wachter, S., Howell, S.B., Ciardi, D.R., et al: 2007, in 15th European Workshop on WDs, R. Napiwotzki \& M. R. Burleigh (eds.), ASP Conf. Ser. 372, 333.

[21] Broggini, C., Bemmerer, D., Guglielmetti, A., Menegazzo, R.: 2010, Annu. Rev. Nucl. Part. Sci. 60, 53-73.

[22] Brown, W.R., Kilic, M., Allende Prieto, C.A., Kenyon, S.J.: 2011, MNRAS, 411, L31.

[23] Cami, J., Bernard-Salas, J., Peeters, E., Malek, S.E.: 2010, Sci., 329, 1180.

[24] Buckley, D.: 2015, talk at the Palermo Workshop on The Golden Age of Cataclysmic Variables and Related Objects - III. 
[25] Cannizzo, J.K., 1993, ApJ, 419, 318.

[26] Cannizzo, J.K., Nelemans, G.: 2015, ApJ 803, 19.

[27] Cassatella, A., Viotti, R. (eds.): 1990, Physics of Classical Novae, Springer-Verlag, Lecture Notes in Physics, 369.

[28] Chadwick, P.M., Dickinson, J.E., Dickinson, M.R., Dipper, N.A., Holder, J. et al.: 1995, APh, 4, 99.

[29] Charles, P.A., Seward, F.D., 1995, in Exploring the Universe, Cambridge University Press, UK, p. 21.

[30] Chiosi, E., Chiosi, C., Trevisan, P., Piovan, L., Orio, M.: 2015, MNRAS 448, 2100.

[31] Churazov, E., Sunyaev, R., Isern, J., Knödlseder, J., Jean, P. et al.: 2014, Nature 512, 406.

[32] Churazov, E., Sunyaev, R., Isern, J., Bikmaev, I., Bravo, E. et al.: 2015, ApJ 812, 62.

[33] Connon Smith, R.: 2007, astro-ph/0701654.

[34] Cordova, F.A.: 1995, in X-Ray Binaries, W. Lewin, J. van Paradijis \& E. van den Heuvel (eds.), Cambridge University Press, p. 331

[35] Cordova, F.A., Mason, K.O.: 1983, in Accretion-Driven Stellar X-Ray Sources, Cambridge University Press, p. 154.

[36] Cordova, F.A., Mason, K.O., Hjellming, R.M.: 1983, PASP, 95, 69

[37] Cordova, F.A., Mason, K.O.: 1984, MNRAS, 206, 879.

[38] Cordova, F.A., Ladd, E.F., Mason, K.O.: 1986, in Magnetospheric Phenomena in Astrophysics, R.I. Epstein \& W.C. Feldman (eds.), AIP, 144, 250.

[39] Cordova, F.A., Howarth, I.D.: 1987, in Exploring the Universe with the IUE Satellite, Y. Kondo (ed.), D. Reidel Publ. Co., Dordrecht, Holland, p. 395.

[40] Craig, N., Abbott, M., Finley, D., Jessop, H., Howell, S.B.,et al.: 1997, ApJS 113, 131.

[41] Darnley, M.J., Ribeiro, V.A.R.M., Bode, M.F., Hounsell, R.A., Williams, R.P.: 2011, arXiv: $1112.2589 \mathrm{v} 1$

[42] Darnley, M.J., Bode, M.F., Harman, D.J., Hounsell, R.A., Munari, U. et al.: 2014, in Stella Novae: Past and Future Decades, P.A. Woudt \& V.A.R.M. Ribeiro (Eds.). ASP Conf. Ser. Vol. 490, 49.

[43] Davis, P.J., Kolb, U., Willems, B., Gänsicke, B.T.: 2008, MNRAS, 389,1563.

[44] la Dous, C.: 1989, A\&A, 211, 131.

[45] la Dous, C.: 1993, in Cataclysmic Variables and Related Objects, M. Hack \& C. la Dous (eds.), NASA SP-507, 15

[46] la Dous, C.: 1994, SSR, 67, 1.

[47] Downes, R.A., Webbink, R.F., Shara, M.M., Ritter, H., Kolb, U., Duerbeck, H.W.: 2001, PASP, 113, 764.

[48] Downes, R.A., Webbink, R.F., Shara, M.M., Ritter, H., Kolb, U., Duerbeck, H.W.: 2006, VizieR On-line Data Catalog: V/123A.

[49] Evans, A., Bode, M.F., O'Brien, T.J., Darnley, M.J. (eds.): 2008, RS Ophiuchi (2006) and the Recurrent Nova Phenomenon, ASP Conf. Ser., Vol. 401.

[50] Evans, A.: 2011, in Asymmetric Planetary Nebulae 5 Conference, A.A. Zijlstra, F. Lykou, I. McDonald, \& E. Lagadec (eds.), Jodrell Bank Centre for Astrophysics, p. 305. 
[51] Fabbiano, G., Hartmann, L., Raymond, J. Steiner, J., Branduardi-Raymond, G., Matilsky, T.: 1981, ApJ, 243, 911.

[52] Ferrario, L., de Martino, D., Gänsicke, B.T.: 2015, SSRv 191, 111-169.

[53] Ferrario, L., Melatos, A., Zrake, J.: 2015, SSRv 191, 77-109.

[54] Forman, W., Jones, C., Cominsky, L., Julien, P., Murray, S., et al.: 1978, ApJS, 38, 357.

[55] Gänsicke, B.T.: 2005, in The Astrophysics of Cataclysmic Variables and Related Objects, J.-M. Hameury \& J.-P. Lasota (Eds.), ASP Conf. Ser. Vol. 330, 3

[56] García-Berro, E., Lorén-Aguilar, P., Aznar-Siguán, G., Torres, S., Camacho, J. et al.: 2012, ApJ 749, 25.

[57] García-Berro, E., Torres, S., Lorén-Aguilar, P., Aznar-Siguán, G., Camacho, J. et al: 2013, in 18th European White Dwarf Workshop, J. Krzesiński, G. Stachowski, P. Moskalik, \& K. Bajan (Eds.), ASP Conf. Proc., Vol. 469, 423.

[58] Gaudenzi, S., Giovannelli, F., Lombardi, R., Claudi, R.: 1986, in New Insights in Astrophysics, E. Rolfe (ed.), ESA SP-263, 455

[59] Gaudenzi, S., Giovannelli, F., Lombardi, R., Claudi, R.: 1990, AcA, 40, 105

[60] Gaudenzi, S., Claudi, R.U., Giovannelli, F., Lombardi, R., Pelosi, M., Strappolini, M.: 2002, in Multifrequency Behaviour of High Energy Cosmic Sources, F. Giovannelli \& L. Sabau-Graziati (eds.), Mem. S.A.It., 73 N. 1, 213.

[61] Gaudenzi S., Giovannelli, F., Mandalari, M., Corradini, M., Lombardi, R.: 2011, A\&A 525, 147.

[62] Giovannelli, F.: 1991, in Frontier Objects in Astrophysics and Particle Physics, F. Giovannelli \& G. Mannocchi (eds.), Italian Physical Society, Editrice Compositori, Bologna, Italy, 28, 3.

[63] Giovannelli, F.: 1996, in Multifrequency Behaviour of High Energy Cosmic Sources, F. Giovannelli \& L. Sabau-Graziati (eds.), Mem. S.A.It., 67, 401.

[64] Giovannelli, F.: 2008, ChJA\&A 8 Suppl., 237.

[65] Giovannelli, F., Gaudenzi, S., Lombardi, R., Bartolini, C., Guarnieri, A., Piccioni, A.: 1985, in Multifrequency Behaviour of Galactic Accreting Sources, F. Giovannelli (ed.), SIDEREA, Roma, Italy, p. 37.

[66] Giovannelli, F., Gonzalez Martinez-Pais, I., Gaudenzi, S., Lombardi, R., Claudi, R.U.: 1990, Ap\&SS $169,125$.

[67] Giovannelli, F., Martinez-Pais, I.G.: 1991, SSR, 56, 313.

[68] Giovannelli, F., Sabau-Graziati, L.: 1998, in Ultraviolet Astrophysics Beyond the IUE Final Archive, W. Wamstecker \& R.Gonzalez-Riestra (eds.), ESA SP-413, 419.

[69] Giovannelli, F., Sabau-Graziati, L.: 1999, in Multifrequency Behaviour of High Energy Cosmic Sources, F. Giovannelli \& L. Sabau-Graziati (eds.), Mem. S.A.It., 70, 987.

[70] Giovannelli, F., Sabau-Graziati, L.: 2004, SSR, 112, 1-443.

[71] Giovannelli, F., Sabau-Graziati, L.: 2012a, in The Golden Age of Cataclysmic Variables and Related Objects, F. Giovannelli \& L. Sabau-Graziati (eds.), Mem. S.A.It., 83 N. 2, 698.

[72] Giovannelli, F., Sabau-Graziati, L.: 2012b, in Second Workshop on Robotic Autonomous Observatories, Bull. Astr. Soc. India (in press). 
[73] Giovannelli, F., Sabau-Graziati, L.: 2012c, Acta Polytechnica, 52 N. 1, 11.

[74] Giovannelli, F., Sabau-Graziati, L.: 2015a, Acta Polytechnica CTU Proc. 2, 3-20.

[75] Giovannelli, F., Sabau-Graziati, L.: 2015b, in Multifrequency Behaviour of High Energy Cosmic Sources - XI, http://pos.sissa.it/cgi-bin/reader/conf.cgi?confid=246, id.1

[76] Godon, P., Sion, E.M.: 2002, ApJ, 566, 1084.

[77] Goliasch, J., Nelson, L.: 2015, ApJ 809, 80.

[78] Greggio, L., Renzini, A., Daddi, E.: 2008, arXiv: 0805.1512v2.

[79] Guy, J., Sullivan, M., Conley, A., Regnault, N., Astier, P., et al.: 2010, A\&A, 523, 7.

[80] Hack, M., la Dous, C.: 1993, in Cataclysmic Variables and Related Objects, M. Hack \& C. la Dous (eds.), NASA SP-507, 1.

[81] Harrison, T.E., McNamara, B.J., Szkody, P., McArthur, B.E., Benedict, G.F. et al.: 1999, ApJL 515, L93.

[82] Hassall, B.J.M., Pringle, J.E., Schwarzenberg-Czerny, A., Wade, R.A., Whelan, J.A.J., Hill, P.W.: 1983, MNRAS, 203, 865.

[83] Hassall, B.J.M., Pringle, J.E., Verbunt, F.: 1985, MNRAS, 216, 353.

[84] Heise, J., Mewe, R., Brinkman, A.C., Gronenschild, E.H.B.M., den Boggende, et al.: 1978, A\&A, 63, L1.

[85] Hellier, C.: 2001, Cataclysmic Variable Stars, Springer

[86] Horne, K., Marsh, T.R., Cheng, F.H., Hubeny, I., Lanz, T.: 1994, ApJ, 426, 294.

[87] Howell, S.B., Cash, J., Mason, K.O., Herzog, A.E.: 1999, AJ 117, 1014.

[88] Howell, S,B., Nelson, L.A., Rappaport, S.: 2001, ApJ 550, 897.

[89] Howell, S.B., Brinkworth, C., Hoard, D.W., Wachter, S., Harrison, T., et al.: 2006, ApJL 646, L65.

[90] Howell, D.A., Conley, A., Della Valle, M., Nugent, P., Perlmutter, S., et al.: 2009, arXiv0903, 1086 H.

[91] Hoyle, F., Fowler, W.A.: 1960, ApJ, 132, 565.

[92] Hudec, R., Šimon, V., Munz, F., Gális, R.: 2008, ChJA\&AS, 8, 381.

[93] Iben, I., Jr., Tutukov, A.V.: 1984, ApJS, 54, 335.

[94] Isern, J., Hernanz, M., García-Berro, E.: 1993, in White Dwarfs: Advances in Observation and Theory, M.A. Barstow (ed.), Kluwer Academic Publ., Dordrecht, Holland, NATO ASI Ser., C403, 139.

[95] Isern, J., Hernanz, M., Abia, C., José, J.: 1997, in Frontier Objects in Astrophysics and Particle Physics, F. Giovannelli \& G. Mannocchi (eds.), Italian Physical Society, Editrice Compositori, Bologna, Italy, 57, 113.

[96] Isern, J., Catalán, S., García-Berro, E., Hernanz, M.: 2013, in 18th European White Dwarf Workshop, J. Krzesiński, G. Stachowski, P. Moskalik, \& K. Bajan (Eds.), ASP Conf. Proc., Vol. 469, 71.

[97] de Jager, O.C., Meintjes, P.J., O’Donoghue, D., Robinson, E.L.: 1994, MNRAS, 267, 577.

[98] Kalomeni, B., Nelson, L., Rappaport, S., Molnar, M., Quintin, J., Yakut, K.: 2016, ApJ 833, 83.

[99] Kepler, S.O., Pelisoli, I., Jordan, S., Kleinman, S.J., Koester, D. et al.:: 2013, MNRAS 429, 2934. 
[100] Kepler, S.O., Pelisoli, I., Koester, D., Ourique, G., Kleinman, S.J. et al.: 2015, MNRAS 446, 4078.

[101] Kilic, M., Brown, W.R., Allende Prieto, C., Agüeros, M.A.; Heinke, C., Kenyon, S.J.: 2011, ApJ, $727,3$.

[102] Kistler, M.D., Stanek, K.Z., Kochanek, C.S., Prieto, J.L., Thompson, T.A.: 2013, ApJ, $770,88$.

[103] Knigge, C.: 2006, MNRAS, 373, 484.

[104] Knigge, C.: 2010, AIPC, 1314, 171.

[105] Knigge, C.: 2011, ASPC, 447, 3.

[106] Knigge, C., Baraffe, I., Patterson, J.: 2011, ApJS, 194, 28.

[107] Körding, E., Rupen, M., Knigge, C., Fender, R., Dhawan, V., et al.: 2008, Science 320, 1318.

[108] Kowalski, M., Rubin, D., Aldering, G, Agostinho, R.J., Amadon, A., et al.: 2008, ApJ, 686, 749.

[109] Landi, R., Bassani, L., Dean, A.J., Bird, A.J., Fiocchi, M. et al.: 2009, MNRAS 392, 630.

[110] Lang, M.J., Buckley, J.H., Carter-Lewis, D.A., Catanese, M., Cawley, M.F. et al.: 1998, APh, 9, 203.

[111] Lasota, J.-P., 2001: New Astron. Rev., 45, 449.

[112] Levitan, D., Groot, P.J., Prince, Th.A., Kulkarni, S.R., Laher, R. et al.: 2015, MNRAS 446, 391.

[113] Lipunov, V.M.: 1987, Ap\&SS, 132, 1.

[114] Lipunov, V.M.: 1991, in Frontier Objects in Astrophysics and Particle Physics, F. Giovannelli \& G. Mannocchi (eds.), Italian Physical Society, Editrice Compositori, Bologna, Italy, 28, 29.

[115] Lira, P.: 1995, Masters thesis, Univ. Chile.

[116] Long, K.S., Mauche, C.W., Raymond, J.C., Szkody, P., Mattei, J.A.: 1996, ApJ, 469, 841

[117] Maguire, K., Sullivan, M., Ellis, R.S., Nugent, P.E., Howell, D.A. et al.: 2012, MNRAS, 426, 2359.

[118] Mannucci, F., Della Valle, M., Panagia, N.: 2006, MNRAS, 370, 773.

[119] Martinez-Pais, I.G., Giovannelli, F., Gaudenzi, S, Rossi, C.: 1996, A\&A, 308, 833.

[120] de Martino, D.: 1998, in Ultraviolet Astrophysics Beyond the IUE Final Archive, W. Wamstecker \& R.Gonzalez-Riestra (eds.), ESA SP-413, 387.

[121] de Martino, D.: 1999, in UV Astronomy in Italy, 1999, L. Buson \& D. de Martino (eds.), Mem. S.A.It., 70, 547.

[122] Mauche, C.W.: 1996, in Cataclysmic Variables and Related Objects, A.Evans \& J.H. Wood (eds.), IAU Coll. N. 158, Kluwer Academic Publishers, p. 243.

[123] Mauche, C.W.: 1998, in Wild Stars in the Old West S. Howell, E. Kuulkers \& C. Woodward (eds.), ASP Conf., 137, 113.

[124] Mauche, C.W., Wade, R.A., Polidan, R.S., van der Woerd, H., Paerels, F.: 1991, ApJ, $372,659$.

[125] Mauche, C.W., Raymond, J.C., Mattei, J.A.: 1995, ApJ, 446, 842.

[126] Meintjes, P.J., Raubenheimer, B.C., de Jager, O.C., Brink, C., Nel, H.I., et al.: 1992, ApJ, $401,325$.

[127] Meintjes, P.J., Oruru, B., Odendaal, A.: 2012, in The Golden Age of Cataclysmic Variables and Related Objects, F. Giovannelli \& L. Sabau-Graziati (eds.), Mem SAIt., 83, 643.

[128] Meyer, F., Meyer-Hofmeister, E.: 1981, A\&A, 104, L10. 
[129] Meyer, F., Meyer-Hofmeister, E.: 1982, A\&A, 106, 34.

[130] Meyer, F., Meyer-Hofmeister, E.: 1983, A\&A, 128, 420.

[131] Mikołajewska, J.: 2011, in Binary Paths in Type Ia Supernovae Explosions, R. Di Stefano \& M. Orio (eds.), IAU Symp. No. 281 (in press). 2011, arXiv: 1110.1847v1.

[132] Mitrofanov, I.G.: 1978, Sov. Astron. Lett., 4, 119.

[133] Mitrofanov, I.G.: 1980, in Close Binary Stars: Observations and Interpretations, D.M. Popper \& R.K. Ulrich (eds.), D. Reidel Publ. Co., Dordrecht, Holland, p. 431.

[134] Mouchet, M., Bonnet-Bidaud, J.M., de Martino, D.: 1998, in Ultraviolet Astrophysics, Beyond the IUE Final Archive, W. Wamstecker \& R. González-Riestra, (eds.), ESA Publication Division, ESTEC, Nordwijk, The Netherlands, ESA SP-413, 431.

[135] Nelemans, G.: 2005, ASPC, 330, 27.

[136] Nelson, L.: 2012, J. Phys.: Conf. Ser. Volume 341, Issue 1, id. 012008.

[137] Norton, A.J., Beardmore, A.P., Allan, A., Hellier, C.: 1999, A\&A, 347, 203.

[138] Norton, A.J., Somerscales, R.V., Wynn, G.A.: 2004, in Magnetic Cataclysmic Variables, S. Vrielmann \&M. Cropper (eds.), IAU Coll. 190, ASP Conf. Ser., 315, 216.

[139] Norton, A.J., Wynn, G.A., Somerscales, R.V.: 2004, ApJ, 614, 349.

[140] Osaki, Y.: 1996, PASP, 108, 390.

[141] Otulakowska-Hypka, M., Olech, A., Patterson, J.: 2016, MNRAS, 460, 2526.

[142] Paczyński, B.: 1967, AcA, 17, 5.

[143] Patat, F., Chandra, P., Chevalier, R., Justham, S., Podsiadlowski, Ph., et al.: 2008, The Messenger, 131,30 .

[144] Patterson, J.: 1979, ApJ, 234, 978.

[145] Patterson, J.: 1984, ApJS, 54, 443.

[146] Patterson, J.: 1992, ApJ 384, 234.

[147] Patterson, J.: 1994, PASP, 106, 209.

[148] Patterson, J.: 1998, PASP, 110, 1132.

[149] Patterson, J.: 2014, in 33rd Annual Symposium on Telescope Science, Society for Astron. Sci., 33, $17-22$.

[150] Patterson, J., Raymond, J.C., 1985, ApJ, 292, 550.

[151] Patterson, J., Uthas, H., Kemp, J., de Miguel, E., Krajci, Th.: 2013, MNRAS, 434, 1902.

[152] Phillips, M.M.: 2005, ASPC, 342, 211.

[153] Phillips, M.M.: 2011, arXiv: 11.4463v1.

[154] Phillips, M.M., Lira, P., Hamuy, M., Maza, J.: 1999, AJ, 118, 1766.

[155] Polidan, R.S., Holberg, J.B.: 1984, Nature 309, 528.

[156] Polidan, R.S., Holberg, J.B.: 1987, MNRAS, 309, 528.

[157] Pretorius, M.L., Warner, B., Woudt, P.A.: 2006, MNRAS, 368, 361. 
[158] Pringle, J.E., Wade, R.A. (eds.): 1985, Interacting Binary Stars, Cambridge Univ. Press.

[159] Rappaport, S., Cash, W., Doxsey, R., McClintock, J., Moore, G.: 1974, ApJ, 187, L5.

[160] Rappaport, S., Verbunt, F., Joss, P.C.: 1983, ApJ, 275, 713.

[161] Ricketts, M.J., King, A.R., Raine, D.J.: 1979, MNRAS, 186, 233.

[162] Reimer, T.W., Welsh, W.F., Mukai, K., Ringwald, F.A.: 2008, ApJ, 678, 376.

[163] Ritter, H.: 1992, in The Astronomy and Astrophysics Encyclopedia, Cambridge University Press, Cambridge, UK, p. 61.

[164] Ritter, H., Kolb, U.: 1998, A\&AS, 129, 83.

[165] Ritter, H., Kolb, U.: 2003, A\&A, 404, 301.

[166] Robinson, E.L.: 1976, ARA\&A, 14, 119.

[167] Rodriguez-Gil, P.: 2003, Ph.D. Thesis, La Laguna University, Spain.

[168] Rodríguez-Gil, P., Gänsicke, B.T., Hagen, H.-J., Araujo-Betancor, S., Aungwerojwit, A., et al.: 2007, MNRAS, 377, 1747.

[169] Scalzo, R.A., Ruiter, A.J., Sim, S.A.: 2014, MNRAS, 445, 2535.

[170] Scaringi, S., Bird, A.J., Norton, A.J., Knigge, C., Hill, A.B. et al.: 2010, MNRAS 401, 2207.

[171] Schaefer, B.E.: 2010, ApJS, 187, 275.

[172] Schaefer, B.E.: 2011, ApJ, 742, 112.

[173] Schmidtobreick, L., Tappert, C.: 2014, in Stella Novae: Past and Future Decades, P.A. Woudt \& V.A.R.M. Ribeiro (Eds.). ASP Conf. Ser. Vol. 490, 29.

[174] Schmidtobreick, L., Tappert, C.: 2015, Acta Polytechnica CTU Proc. Vol. 2, 188.

[175] Seitter, W.C..: 1990, in Physics of Classical Novae, A. Cassatella \& R. Viotti (eds.), Springer-Verlag, Lecture Notes in Physics, 369, 79.

[176] Shafter, A.W., Curtin, C., Pritchet, C.J., Bode, M.F., Darnley, M.J.: 2014, in Stella Novae: Past and Future Decades, P.A. Woudt \& V.A.R.M. Ribeiro (eds.), ASP Conf. Ser., 490, 77.

[177] Shafter, A.W., Henze, M., Rector, T.A., Schweizer, F., Hornoch, K. et al.: 2015, ApJS, 216, 34.

[178] Shakura, N.I., Sunyaev, R.A.: 1973, A\&A, 24, 337.

[179] Shen, K.J.: 2015, ApJL 805, L6.

[180] Sidro, N., Cortina, J., Mauche, C.W., de Oña, E., Torres, D.F.: 2008, ICRC. 2, 715.

[181] Šimon, V., Hudec, R., Munz, F., Štrobl, J.: 2006, ChJA\&A, 6 Suppl. 1, 149.

[182] Sion, E.M.: 1986, PASP, 98, 821.

[183] Sion, E.M.: 1991, AJ, 102, 295.

[184] Smak, J.: 1967, AcA, 17, 3.

[185] Smak, J.: 1984a, AcA, 34, 161.

[186] Smak, J.: 1984b, PASP, 96, 5.

[187] Smak, J.: 1985a, in Multifrequency Behaviour of Galactic Accreting Sources, F. Giovannelli (ed.), SIDEREA, Roma, Italy, p. 3. 
[188] Smak, J.: 1985b, in Multifrequency Behaviour of Galactic Accreting Sources, F. Giovannelli (ed.), SIDEREA, Roma, Italy, p. 17.

[189] Smak, J.: 2002, in Multifrequency Behaviour of High Energy Cosmic Sources, F. Giovannelli \& L. Sabau-Graziati (eds.), Mem. S.A.It., 73 N. 1, 206.

[190] Solheim, J.-E.: 2010, PASP, 122, 1133-1163.

[191] Stril, A., Cahn, R.N., Linder, E.V.: 2010, MNRAS, 404, 239.

[192] Surina, F., Bode, M.F., Darnley, M.J: 2011, arXiv: 1111.5524v1.

[193] Surina, F., Bode, M.F., Darnley, M.J.: 2015, PKAS, 30, 237.

[194] Szkody, P.: 1998, in Ultraviolet Astrophysics, Beyond the IUE Final Archive, W. Wamstecker \& R. González-Riestra, (eds.), ESA Publication Division, ESTEC, Nordwijk, The Netherlands, ESA SP-413, 381.

[195] Szkody, P., Gänsicke, B.T.: 2012, JAAVSO 40, 563.

[196] Terada, Y., Ishida, M., Bamba, A., Mukai, K., Hayashi, T., Harayama, A.: 2010, ApJ, 721, 1908.

[197] Townsley, D.M., Bildsten, L.: 2005, ApJ, 628, 395.

[198] Tsebrenko, D., Soker, N.: 2015, MNRAS, 447, 2568.

[199] Urban, J.A., Sion, E.M.: 2006, ApJ, 642, 1029.

[200] Verbunt, F.: 1987, A\&AS, 71, 339.

[201] Verbunt, F., Hassall, B.M.J., Pringle, J.E., Warner, B., Marang, F.: 1987, MNRAS, 225, 113.

[202] Verbunt, F., Bunk, W.H., Ritter, H., Pfeffermann, E.: 1997, A\&A, 327, 602.

[203] Voss, R., Nelemans, G.: 2008, Nature, 451, 802.

[204] Vrielmann, S., Cropper, M. (eds.): 2004, in Magnetic Cataclysmic Variables, IAU Colloquium 190, ASP Conf. Proc. Vol. 315. San Francisco: Astronomical Society of the Pacific.

[205] Warner, B.: 1976, The Observatory, 96, 49.

[206] Warner, B.: 1987, MNRAS, 227, 23.

[207] Warner, B.: 1995a, Cataclysmic Variable Stars, Cambridge Astrophysics Series, 28, Cambridge University Press.

[208] Warner, B.: 1995b, in Magnetic cataclysmic variables, D.A.H. Buckley \& B. Warner (eds.), ASP Conf. Ser., 85, 3.

[209] Warner, B.: 1995c, Ap\&SS, 225, 249.

[210] Warner, B.: 1996, Ap\&SS 241, 263.

[211] Warner, B.: 2001, in Small Telescope Astronomy on Global Scales, W.-P. Chen, C. Lemme, \& B. Paczynski (eds.), IAU Coll. 183, ASP Conf. Ser., 246, 159.

[212] Warner, B.: 2002, in Classical Nova Explosions, AIP Conf. Proc., 637, 3.

[213] Warner, B.: 2004, PASP, 116, 115.

[214] Warner, B., Wickramasinghe, D.T.: 1991, MNRAS, 248, 370.

[215] Warner, B., Woudt, P.A.: 2005, in The Astrophysics of Cataclysmic Variables and Related Objects, J.-M. Hameury \& J.-P. Lasota (eds.), ASP Conf. Ser., 330, 227. 
[216] Watson, M.G., King, A.R., Heise, J.: 1985, SSR, 40, 127.

[217] Webbink, R.F.: 1984, ApJ, 277, 355.

[218] Wheatley, P.J., Mauche, C.W., Mattei, J.A.: 2003, MNRAS, 345, 49.

[219] Whelan, J., Iben, I. Jr.: 1973, ApJ, 186, 1007.

[220] Wickramasinghe, D.T., Ferrario, L.: 2000, PASP 112, Issue 773, 873-924.

[221] Willems, B., Kolb, U., Sandquist, E.L., Taam, R.E., Dubus, G.: 2005, ApJ, 635, 1263.

[222] Willems, B., Taam, R.E, Kolb, U., Dubus, G., Sandquist, E.L.: 2007, ApJ, 657, 465.

[223] Williams, S.C., Darnley, M.J., Bode, M.F., Shafter, A.W.: 2014, in Stella Novae: Past and Future Decades, P.A. Woudt \& V.A.R.M. Ribeiro (eds.), ASP Conf. Ser., 490, 85.

[224] Williams, S.C., Darnley, M.J., Bode, M.F., Shafter, A.W.: 2016, ApJ. 817. 143.

[225] Witham, A.R., Knigge, C., Aungwerojwit, A., Drew, J.E., Gänsicke, B.T., et al.: 2007, MNRAS, $382,1158$.

[226] Wood, J.H., Horne, K., Berriman, G., Wade, R.A.: 1989, ApJ, 341, 974.

[227] Woudt, P.A., Warner, B.: 2003, MNRAS, 340, 101.

[228] Woudt, P.A., Warner, B., Pretorius, M.L.: 2004, MNRAS, 351, 1015.

[229] Woudt, P.A., Warner, B., Spark, M.: 2005, MNRAS, 364, 107.

[230] Wynn, G.A.: 2000, New Astr. Rev., 44, 75.

[231] Wynn, G.A., King, A.R., Horne, K.: 1997, MNRAS, 286, 436.

[232] Yaron, O., Prialnik, D., Shara, M.M., Kovetz, A.: 2005, ApJ, 623, 398.

[233] Yoon, S.-C., Podsiadlowski, Ph., Rosswog, S.: 2007, MNRAS, 380, 933.

[234] Zhang, E., Robinson, E.L., Stiening, R.F., Horne, K.: 1995, ApJ, 454, 447. 